TITLE:

\title{
Quenched tail estimate for the random walk in random scenery and in random layered conductance
}

\author{
$\operatorname{AUTHOR}(\mathrm{S})$ :
}

Deuschel, Jean-Dominique; Fukushima, Ryoki

\section{CITATION:}

Deuschel, Jean-Dominique ... [et al]. Quenched tail estimate for the random walk in random scenery and in random layered conductance. Stochastic Processes and their Applications 2019, 129(1): 102-128

ISSUE DATE:

2019-1

URL:

http://hdl.handle.net/2433/241736

\section{RIGHT:}

(c) 2019. This manuscript version is made available under the CC-BY-NC-ND 4.0 license

http://creativecommons.org/licenses/by-nc-nd/4.0/; The full-text file will be made open to the public on 1 January 2021 in accordance with publisher's 'Terms and Conditions for Self-Archiving'.; This is not the published version. Please cite only the published version.; この論文は出版社版でありません。引用の際には出版社版をご確認ご利用ください。 


\title{
QUENCHED TAIL ESTIMATE FOR THE RANDOM WALK IN RANDOM SCENERY AND IN RANDOM LAYERED CONDUCTANCE
}

\author{
JEAN-DOMINIQUE DEUSCHEL AND RYOKI FUKUSHIMA
}

\begin{abstract}
We discuss the quenched tail estimates for the random walk in random scenery. The random walk is the symmetric nearest neighbor walk and the random scenery is assumed to be independent and identically distributed, nonnegative, and has a power law tail. We identify the long time asymptotics of the upper deviation probability of the random walk in quenched random scenery, depending on the tail of scenery distribution and the amount of the deviation. The result is in turn applied to the tail estimates for a random walk in random conductance which has a layered structure.
\end{abstract}

\section{INTRODUCTION}

In this article, we study the random walk in random scenery, mainly in the continuous time setting. This model is first introduced and studied in the discrete time setting by Borodin [7, 8] and Kesten-Spitzer [26] independently. It is the sum of independent and identically distributed random variables $\left(\{z(x)\}_{x \in \mathbb{Z}^{d}}, \mathbb{P}\right)$ along a random walk $\left(\left(S_{n}\right)_{n \in \mathbb{Z}_{+}}, P_{0}\right)$ starting at the origin:

$$
W_{n}=\sum_{k=1}^{n} z\left(S_{k}\right) .
$$

One of the motivation in [26] was to construct a new class of self-similar processes as a scaling limit of this process under the joint law. They proved that, when $d=1$ and $z$ and $S$ are centered and belong to the domain of attraction of stable law with index $\alpha \in(0,2]$ and $\beta \in(1,2]$ respectively, the rescaled process

$$
\left(n^{-\left(1-\frac{1}{\beta}+\frac{1}{\alpha \beta}\right)} W_{\lceil n t\rceil}\right)_{t \geq 0}
$$

converges in distribution under $\mathbb{P} \otimes P_{0}$. Subsequently, a lot of works have been done to extend the distributional limit theorems, not only under the joint law but also for the quenched scenery, namely for almost all realizations of the scenery, and to obtain law of iterated logarithm type results. We refer the reader to the introduction of [24] for more details and background.

Date: February 13, 2019.

2010 Mathematics Subject Classification. Primary 60F10; secondary 60J55; 60K37.

Key words and phrases. random walk, random scenery, tail estimate, moderate deviation, large deviation, random conductance model, layered media. 
In this paper, we discuss upper tail estimates with quenched scenery which is assumed to be non-negative and have a power law tail. The tail estimate for the random walk in random scenery is studied rather recently. As for the annealed tail estimates, that is, under the joint law, there are extensive results. In the case of Gaussian scenery, for the Brownian motion [1]] and for the Markov chains satisfying the level-2 full large deviation principle [2:3] instead of the random walk, the large deviation principles for $t^{-\frac{3}{2}} W_{t}$ and $n^{-\frac{3}{2}} W_{n}$ are proved. Later the moderate deviations are also studied for the Brownian motion in $d \leq 3$ [10]. The paper [28] also includes an upper tail estimate for the stable process in Brownian scenery, which is a continuous space counterpart. The bounded scenery case is studied in [3], 2] for the Brownian motion and the large deviation principle for $t^{-1} W_{t}$ is established. More recently, the random walk in a random scenery with stretched or compressed exponential tail attracted much attention, partially in relation to the recent development on the tail estimates for the self-intersection local time [13., 2.9]. There are various results depending on the tail and the spatial dimensions [4, 5, 201, 21]. Later, under the Cramér condition for the upper tail and the finiteness of third moment, [1.9] proved quite precise moderate deviation estimates. Also in one dimensional case, the moderate deviations are studied for stable random walk in a scenery with sub-Gaussian tail [30] $]$.

Concerning the quenched tail estimates, there are not many results. In the case of one dimensional Brownian motion in Gaussian scenery [3] and bounded scenery [2], the large deviation principles are proved for $t^{-1}(\log t)^{-\frac{1}{2}} W_{t}$ and $t^{-1} W_{t}$ respectively. And again [I0] extends the result to the moderate deviations for the Brownian motion in Gaussian scenery for $d \leq 3$. For more on the technical correspondence, see Remark $\nabla$ below. One may also consider the tail behavior under the scenery law with the random walk trajectory fixed. In this case the large deviation principle is proved in [23].

The random walk in random scenery is naturally interpreted as a diffusing particle in a layered random media. See for example the introduction of [3] where it is studied under the name "diffusion in a random Gaussian shear flow drift". In this paper, we study another model - the random walk in a random layered conductance introduced and studied in [I]. Here again we assume that the random conductance has a power law tail. See Section 2.2 below for the precise setting. This process can be described as the time change of the simple random walk with the clock process given by the random walk in random scenery. By using the tail estimates for the latter, we derive asymptotics of the super-diffusive deposition probabilities.

\section{Setting AND ReSUlts}

Throughout the paper, $\left(\{z(x)\}_{x \in \mathbb{Z}^{d}}, \mathbb{P}\right)$ is a family of non-negative, independent and identically distributed random variables with the distribution satisfying

$$
\mathbb{P}(z(x)>r)=r^{-\alpha+o(1)} \text { as } r \rightarrow \infty
$$


for some $\alpha>0$.

Remark 1. The non-negativity could be replaced by some assumption on the lower tail. For instance, $z$ being bounded from below would suffice for the results in Section [.]. We work with non-negative scenery with an application to the random conductance model (Section [2.2) in mind.

2.1. Random walk in random scenery. Let $\left(\left\{S_{t}\right\}_{t \geq 0}, P_{x}\right)$ be the continuous time simple random walk on $\mathbb{Z}^{d}$ starting at $x \in \mathbb{Z}^{d}$. We consider the additive functional defined by

$$
A_{t}=\int_{0}^{t} z\left(S_{u}\right) \mathrm{d} u
$$

This is a natural continuous time analogue of the random walk in random scenery.

Theorem 1. Let $\rho>0$. Then $\mathbb{P}$-almost surely,

$$
P_{0}\left(A_{t} \geq t^{\rho}\right)=\exp \left\{-t^{p(\alpha, \rho)+o(1)}\right\}
$$

as $t \rightarrow \infty$, where

$$
p(\alpha, \rho)= \begin{cases}\frac{2 \alpha \rho}{\alpha+1}-1, & \rho \in\left(\frac{\alpha+1}{2 \alpha} \vee 1, \frac{\alpha+1}{\alpha}\right] \\ \alpha(\rho-1), & \rho>\frac{\alpha+1}{\alpha}\end{cases}
$$

for $d=1$ and

$$
p(\alpha, \rho)= \begin{cases}\frac{2 \alpha \rho-d}{2 \alpha+d}, & \rho \in\left(\frac{d}{2 \alpha} \vee 1, \frac{\alpha+d}{\alpha}\right], \\ \frac{\alpha(\rho-1)}{d}, & \rho>\frac{\alpha+d}{\alpha}\end{cases}
$$

for $d \geq 2$. Furthermore, when $d=1$ and $\rho<\frac{\alpha+1}{2 \alpha} \vee 1$ or $d \geq 2$ and $\rho<\frac{d}{2 \alpha} \vee 1$, $\mathbb{P}$-almost surely the above probability is bounded from below by a negative power of $t$.

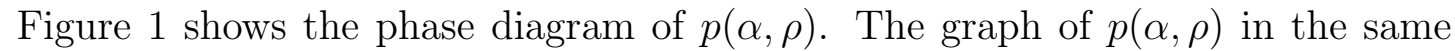
case can be found in Figures $\mathbb{\theta}$ and 5 in subsection 5.1.

Remark 2. We can prove the corresponding results for the discrete time random walk in random scenery by the same argument. The exponent in the first regime is the same but in the second regime it would be $\infty$. This is because the random walk needs to travel much further distance than $t$ to achieve $A_{t} \geq t^{\rho}$ in the second regime. See Section 42 below.

Corollary 1. Let $\alpha \leq 1$. Then $\mathbb{P} \otimes P_{0}$-almost surely,

$$
\limsup _{t \rightarrow \infty} \frac{\log A_{t}}{\log t} \leq \begin{cases}\frac{\alpha+1}{2 \alpha}, & d=1 \\ \frac{d}{2 \alpha}, & d \geq 2 .\end{cases}
$$




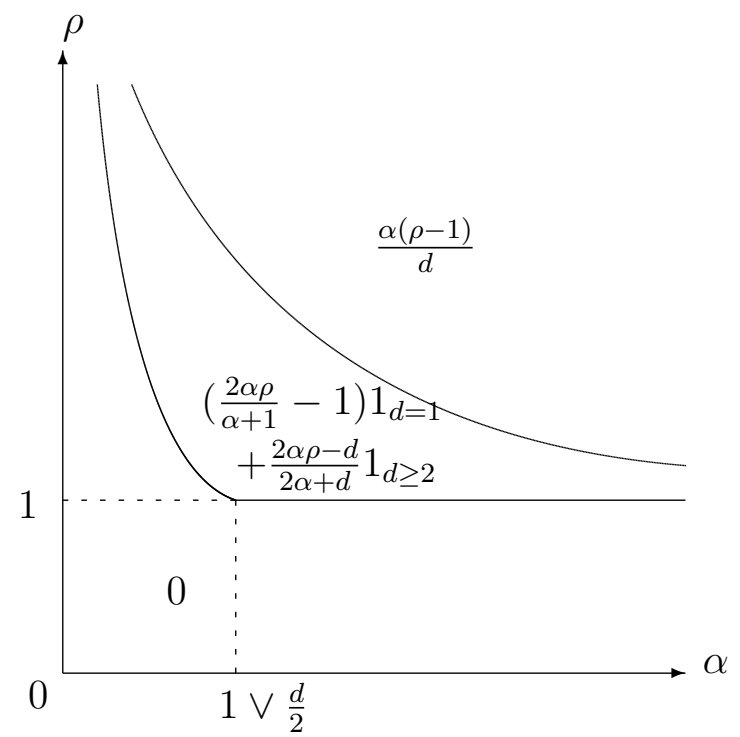

Figure 1 . The phase diagram of the exponent $p(\alpha, \rho)$. The curves are $\rho=\left(\frac{\alpha+1}{2 \alpha} 1_{d=1}+\frac{d}{2 \alpha} 1_{d \geq 2}\right) \vee 1$ and $\rho=\frac{\alpha+d}{\alpha}$.

Remark 3. This follows immediately from Theorem $\square$ and the Borel-Cantelli Lemma and is, to the best of our knowledge, new. The exponent in the one dimensional case coincides with the self-similar parameter identified in [26], see ([י2). When $d \geq 2$, the random walk visits a single site not too many times and hence $A_{t}$ should behave in a similar way to the sum of independent and identically distributed random variables, in which case the scaling exponent is $1 / \alpha$. Based on this observation, we believe that ([2.6) is not sharp for $d \geq 3$. We focus on $\alpha \leq 1$ since in the other case $\alpha>1$, one can prove much finer asymptotics

$$
\lim _{t \rightarrow \infty} \frac{1}{t} A_{t}=\mathbb{E}[z(0)]
$$

$\mathbb{P} \otimes P_{0}$-almost surely, for example by using Kakutani's random ergodic theorem [25] as pointed out in [17]. In one-dimensional case with stronger moment conditions, even finer asymptotics, the law of iterated logarithm for $A_{t}-t \mathbb{E}[z(0)]$, is shown in [14, 34].

When $d=1, \alpha \leq 1$ and $\rho=\frac{\alpha+1}{2 \alpha}$, the bound (2.3) with $p(\alpha, \rho)=0$ holds by monotonicity. The same applies also for $d \geq 2, \alpha \leq \frac{d}{2}$ and $\rho=\frac{d}{2 \alpha}$. On the other hand, when $d=1, \alpha>1$ and $\rho=1$ or $d \geq 2, \alpha>\frac{d}{2}$ and $\rho=1$, the tail of $P_{0}\left(A_{t} \geq c t\right)$ depends on $c$. This tends to one for $c<\mathbb{E}[z(0)]$ whereas for $c>\mathbb{E}[z(0)]$, this is the standard large deviation regime. We can get a lower bound by extrapolation and in fact it is the correct tail behavior. 
Theorem 2. Let $d=1$ and $\alpha>1$ or $d \geq 2$ and $\alpha>\frac{d}{2}$. Then for any $c>\mathbb{E}[z(0)]$, $\mathbb{P}$-almost surely,

$$
P_{0}\left(A_{t} \geq c t\right)= \begin{cases}\exp \left\{-t^{\frac{\alpha-1}{\alpha+1}+o(1)}\right\}, & d=1, \\ \exp \left\{-t^{\frac{2 \alpha-d}{2 \alpha+d}+o(1)}\right\}, & d \geq 2\end{cases}
$$

as $t \rightarrow \infty$.

2.2. Random walk in layered random conductance. The model considered here is the random walk in random conductance in $\mathbb{Z}^{1+d}$ which is constant along lines parallel to an axis. We write $x \in \mathbb{Z}^{1+d}$ as $\left(x_{1}, x_{2}\right)$ with $x_{1} \in \mathbb{Z}$ and $x_{2} \in \mathbb{Z}^{d}$. In what follows, any points in $\mathbb{R}^{k} \backslash \mathbb{Z}^{k}(k \in \mathbb{N})$ are to be understood as closest lattice points. Let $\left(\left(X_{t}\right)_{t \geq 0}, P_{x}^{\omega}\right)$ be a continuous time Markov chain with jump rates

$$
\omega\left(x, x \pm \mathbf{e}_{i}\right)= \begin{cases}z\left(x_{2}\right), & i=1, \\ 1, & i \geq 2 .\end{cases}
$$

Such a process is sometimes called the variable speed random walk in the random conductance field $\omega$. This is related to the random walk in random scenery as follows: Let $\left(S^{1}, S^{2}\right)$ be the continuous time simple random walk on $\mathbb{Z}^{1+d}$. Then we have a representation

$$
\left(X_{t}^{1}, X_{t}^{2}\right)_{t \geq 0}=\left(S_{A_{t}^{2}}^{1}, S_{t}^{2}\right)_{t \geq 0},
$$

where the clock process is defined by $A_{t}^{2}=\int_{0}^{t} z\left(S_{u}^{2}\right) \mathrm{d} u$.

This model is introduced in [I], Example 1.11 in order to demonstrate an anomalous tail behavior of the transition probability and an upper bound is obtained. Define a random distance - sometimes called the chemical distance - by

$$
d^{\omega}(x, y)=\inf _{\gamma \in \Gamma(x, y)} \sum_{e \in \gamma} \frac{1}{\sqrt{\omega(e)} \vee 1},
$$

where $\Gamma(x, y)$ denotes the set of nearest neighbor paths connecting $x$ and $y$. Then the following upper bound is proved in [I] , Theorem 1.10:

$$
P_{x}^{\omega}\left(X_{t}=y\right) \leq c t^{-\frac{d}{2}} \begin{cases}\exp \left\{-c^{-1} \frac{d^{\omega}(x, y)^{2}}{t}\right\}, & t>d^{\omega}(x, y), \\ \exp \left\{-c^{-1} d^{\omega}(x, y)\left(1 \vee \log \frac{d^{\omega}(x, y)}{t}\right)\right\}, & t \leq d^{\omega}(x, y) .\end{cases}
$$

We take $x=0, y=t^{\delta} \mathbf{e}_{1}+t^{\gamma} \mathbf{e}$ with $\gamma \geq 0, \delta \in\left(\frac{1}{2}, \infty\right)$, and e being a unit vector orthogonal to $\mathbf{e}_{1}$, that is, we are looking at a super-diffusive deposition. It is not difficult to show that

$$
d^{\omega}\left(0, t^{\delta} \mathbf{e}_{1}+t^{\gamma} \mathbf{e}\right)=t^{\frac{2 \delta \alpha}{2 \alpha+\alpha} \vee \gamma+o(1)}, \text { as } t \rightarrow \infty
$$

$\mathbb{P}$-almost surely (see Lemma 1.12 in [T] for the case $d=1$ ). Then (2.I2) yields a stretched-exponential upper bound

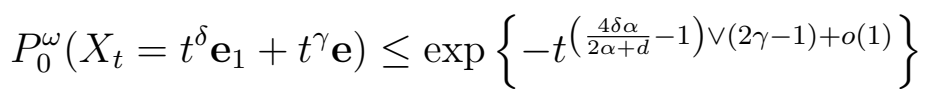


when $\frac{2 \delta \alpha}{2 \alpha+d} \vee \gamma \leq 1$. We are interested in whether this captures the correct asymptotics. The following theorem shows that it is sharp if and only if $\delta=\frac{2 \alpha+d}{2 \alpha}$ and $\gamma \leq 1$. Note that in this case, we have $d^{\omega}\left(0, t^{\delta} \mathbf{e}_{1}+t^{\gamma} \mathbf{e}\right)=t^{1+o(1)}$ which corresponds to the boundary case of $(2.12)$.

Theorem 3. Let $\gamma, \delta \geq 0$ and $\mathbf{e}$ be a unit vector orthogonal to $\mathbf{e}_{1}$. Then for $\mathbb{P}$-almost every $\omega$,

$$
P_{0}^{\omega}\left(X_{t}=t^{\delta} \mathbf{e}_{1}+t^{\gamma} \mathbf{e}\right)=\exp \left\{-t^{q(\alpha, \delta) \vee((2 \gamma-1) \wedge \gamma)+o(1)}\right\}
$$

as $t \rightarrow \infty$, where with the convention $\frac{\alpha}{0}=\infty$, for $d=1$,

$$
q(\alpha, \delta)= \begin{cases}0, & \delta<\frac{1}{2} \vee \frac{\alpha+1}{4 \alpha}, \\ 2 \delta-1, & \delta \in\left[\frac{1}{2}, \frac{\alpha}{\alpha+1}\right), \\ \frac{4 \alpha \delta-\alpha-1}{3 \alpha+1}, & \delta \in\left[\frac{\alpha}{\alpha+1} \vee \frac{\alpha+1}{4 \alpha}, \frac{2 \alpha+1}{2 \alpha}\right], \\ \frac{\alpha(2 \delta-1)}{\alpha+1}, & \delta \in\left(\frac{2 \alpha+1}{2 \alpha}, \frac{\alpha}{(\alpha-1)_{+}}\right), \\ \delta, & \delta \geq \frac{\alpha}{(\alpha-1)_{+}} .\end{cases}
$$

The second case is to be understood as void for $\alpha<1$. For $d \geq 2$,

$$
q(\alpha, \delta)= \begin{cases}0, & \delta<\frac{1}{2} \vee \frac{d}{4 \alpha}, \\ 2 \delta-1, & \delta \in\left[\frac{1}{2}, \frac{2 \alpha}{2 \alpha+d}\right), \\ \frac{4 \alpha \delta-d}{4 \alpha+d}, & \delta \in\left[\frac{2 \alpha}{2 \alpha+d} \vee \frac{d}{4 \alpha}, \frac{2 \alpha+d}{2 \alpha}\right], \\ \frac{\alpha(2 \delta-1)}{\alpha+d}, & \delta \in\left(\frac{2 \alpha+d}{2 \alpha}, \frac{\alpha}{(\alpha-d)_{+}}\right), \\ \delta, & \delta \geq \frac{\alpha}{(\alpha-d)_{+}} .\end{cases}
$$

with the second case understood as void for $\alpha<\frac{d}{2}$.

Theorem 4. Let $\gamma \leq \frac{1}{2}$ and $\mathbf{e}$ be a unit vector orthogonal to $\mathbf{e}_{1}$. Suppose $d=1$ and $\delta<\frac{1}{2} \vee \frac{\alpha+1}{4 \alpha}$ or $d \geq 2$ and $\delta<\frac{1}{2} \vee \frac{d}{4 \alpha}$. Then, $\mathbb{P}$-almost surely, $P_{0}^{\omega}\left(X_{t}=t^{\delta} \mathbf{e}_{1}+t^{\gamma} \mathbf{e}\right)$ is bounded from below by a negative power of $t$.

Figures 2 and 3 show the phase diagrams of the displacement exponents $q(\alpha, \delta) \vee$ $((2 \gamma-1) \wedge \gamma)$ in the one-dimensional case.

In the heat kernel estimate for random walks in random environment, it is sometimes useful to give a probabilistic control on the range of time (or space) in which an estimate like Theorem 3 holds true. It turns out that we cannot hope much in this model. For $r>0$, define

$$
\tau_{r}=\sup \left\{t \geq 0:\left|\frac{1}{\log t} \log \right| \log P_{0}^{\omega}\left(X_{t}=t^{\delta} \mathbf{e}_{1}+t^{\gamma} \mathbf{e}\right)|-q(\alpha, \delta)|>r\right\} .
$$

that is, the last time when the upper or lower bound is violated by $r$ in the exponent. 


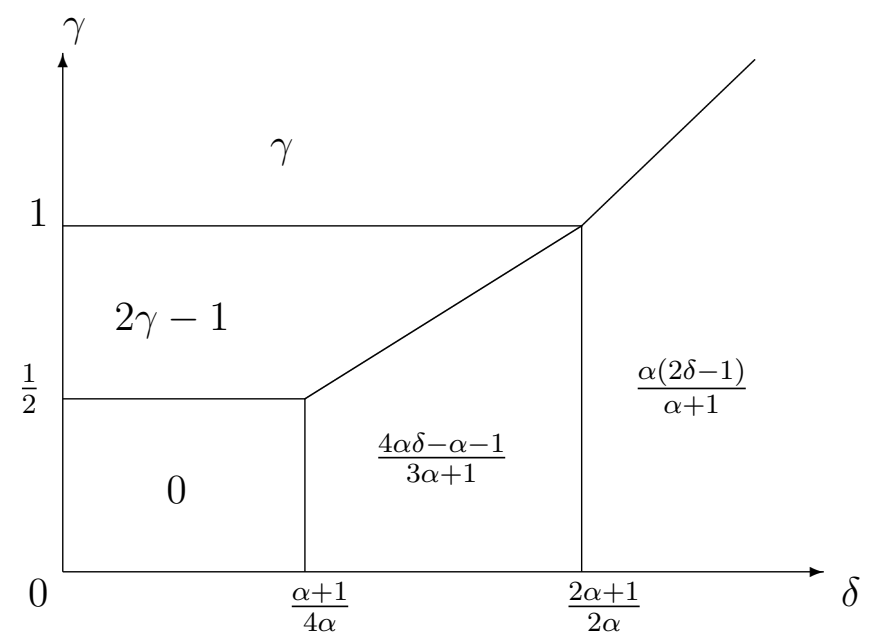

Figure 2. The phase diagram of the displacement exponents in the case $d=1$ and $\alpha \leq 1$. The slopes of the increasing piecewise linear curve starting at $\left(\frac{\alpha+1}{4 \alpha}, \frac{1}{2}\right)$ are $\frac{2 \alpha}{3 \alpha+1}$ and $\frac{2 \alpha}{\alpha+1}$ from the left.

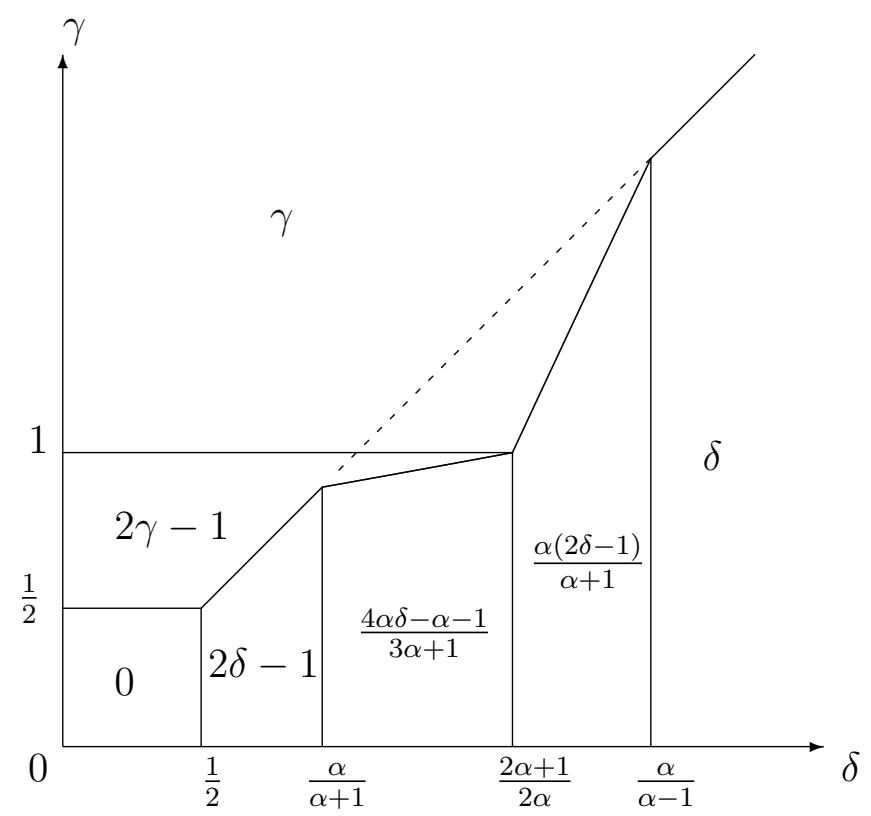

Figure 3. The phase diagram of the displacement exponents in the case $d=1$ and $\alpha>1$. The slopes of the increasing piecewise linear curve starting at $\left(\frac{1}{2}, \frac{1}{2}\right)$ are $1, \frac{2 \alpha}{3 \alpha+1}, \frac{2 \alpha}{\alpha+1}$ and 1 from the left.

Theorem 5. Suppose $q(\alpha, \delta)>(2 \gamma-1) \wedge \gamma$. Then in the third or fourth regimes in ([.]6) and ([.]7), for sufficiently small $r>0$,

$$
\mathbb{P}\left(\tau_{r} \geq t\right) \geq t^{-r\left(C_{1}+o(1)\right)}
$$


as $t \rightarrow \infty$, where

$$
C_{1}= \begin{cases}\frac{\alpha+1}{2}, & d=1 \text { and } \delta \in\left(\frac{\alpha}{\alpha+1} \vee \frac{\alpha+1}{4 \alpha}, \frac{2 \alpha+1}{2 \alpha}\right] \\ \alpha+\frac{d}{2}, & d \geq 2 \text { and } \delta \in\left(\frac{2 \alpha}{2 \alpha+d} \vee \frac{d}{4 \alpha}, \frac{2 \alpha+d}{2 \alpha}\right], \\ d, & d \geq 1 \text { and } \delta \in\left(\frac{2 \alpha+d}{2 \alpha}, \frac{\alpha}{(\alpha-d)_{+}}\right) .\end{cases}
$$

In the second regime in ([.]6) and ([2.T7), for sufficiently small $r>0$,

$$
\mathbb{P}\left(\tau_{r} \geq t\right) \geq \begin{cases}t^{\left.-\alpha-\delta(\alpha-1)-\frac{r}{2}(3 \alpha+1)\right)+o(1)}, & d=1, \\ t^{\left.-2 \alpha+\delta(2 \alpha+d)-\frac{r}{2}(4 \alpha+d)\right)+o(1)}, & d \geq 2 .\end{cases}
$$

Remark 4. We only stated the lower bounds since the point of Theorem is the slow decay of $\mathbb{P}\left(\tau_{r} \geq t\right)$. With some additional effort, it is possible to find the

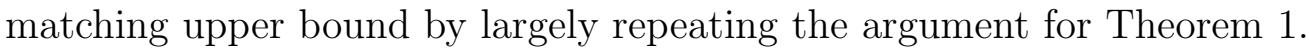

Remark 5. We exclude the case $q(\alpha, \delta) \leq(2 \gamma-1) \wedge \gamma$ since there the heat kernel bound has little to do with $z$-field. In particular, the only possibility is that the lower bound is violated and it must be because of atypically small values of $z$. Based on this observation, one can show that for sufficiently small $r, \mathbb{P}\left(\tau_{r} \geq t\right)$ is bounded by $\exp \left\{-t^{C_{2}}\right\}$ for some $C_{2}>0$.

\section{A BOUND ON THE CONTINUOUS TIME RANDOM WALK}

We frequently use the following estimate on the transition probability of the continuous time simple random walk $p_{t}(x, y)=P_{x}\left(S_{t}=y\right)$. This can be found in [16], Proposition 4.2 and 4.3 .

Lemma 1. There exist positive constants $c_{1}-c_{4}$ such that when $t \geq 1$,

$$
c_{1} t^{-\frac{d}{2}} \exp \left\{-c_{2} \frac{|x|^{2}}{t}\right\} \leq p_{t}(0, x) \leq c_{3} t^{-\frac{d}{2}} \exp \left\{-c_{4} \frac{|x|^{2}}{t}\right\}
$$

for $|x| \leq t$ and

$$
\exp \left\{-c_{2}|x|\left(1 \vee \log \frac{|x|}{t}\right)\right\} \leq p_{t}(0, x) \leq \exp \left\{-c_{4}|x|\left(1 \vee \log \frac{|x|}{t}\right)\right\}
$$

for $|x|>t$.

\section{PROOFS FOR THE RANDOM WALK IN RANDOM SCENERY}

Both for the lower and upper bounds, we shall restrict our attention to the event

$$
\left\{\max _{0 \leq u \leq t}\left|S_{u}\right| \leq t^{\mu}\right\}
$$

for a certain $\mu \geq \frac{1}{2}$. Note that $\mathbb{P}$-almost surely,

$$
\max _{|x| \leq t^{\mu}} z(x)=t^{\frac{d \mu}{\alpha}+o(1)}
$$


and the point of maximum $x_{\max }$ of $z$ within $|x| \leq t^{\mu}$ satisfies $\left|x_{\max }\right|=t^{\mu+o(1)}$ as $t \rightarrow \infty$.

4.1. Lower bounds of Theorem $\mathbf{T}$. We prove the lower bounds for the probability with an additional terminal constraint

$$
P_{0}\left(A_{t} \geq t^{\rho}, S_{t}=0\right) \geq \exp \left\{-t^{p(\alpha, \rho)+o(1)}\right\},
$$

with the right hand side replaced by a negative power of $t$ when $p(\alpha, \rho)=0$, i.e., $\rho<$ $\frac{\alpha+1}{2 \alpha} \vee 1(d=1)$ or $\rho<\frac{d}{2 \alpha} \vee 1(d \geq 2)$. We will use these bounds in the proof of

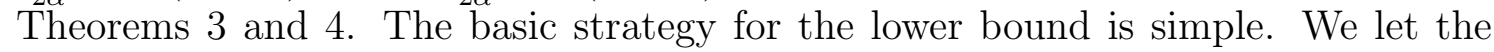
random walk go to $x_{\max }$ within time $\frac{t}{4}$, leave the right amount of local time there by the time $\frac{t}{2}$ and then come back to the origin. In the second regime where $\rho>\frac{\alpha+d}{\alpha}$, the random walk stays at $x_{\max }$ all the time in $\left[\frac{t}{4}, \frac{t}{2}\right]$ and its probability is easy to evaluate. But in the other regime, the optimal strategy is to leave the local time much smaller than $\frac{t}{4}$ and we need a moderate deviation estimate for the local time in [I:2]. Throughout the proof of the lower bound, we assume (4.2).

The SECOND REgIME $\rho>\frac{\alpha+d}{\alpha}$ : In this case, we choose $\mu=\frac{\alpha(\rho-1)}{d}+\epsilon>1$ so that $\rho<\frac{d \mu}{\alpha}+1$. Then the above strategy of the random walk yields $A_{t} \geq \frac{1}{4} t^{\frac{d \mu}{\alpha}+1}>t^{\rho}$. By using the Markov property and Lemma $\square$, we have

$$
\begin{aligned}
& P_{0}\left(A_{t} \geq t^{\rho}, S_{t}=0\right) \\
& \geq P_{0}\left(S_{\frac{t}{4}}=x_{\max }\right) P_{x_{\max }}\left(S_{u}=x_{\max } \text { for any } u \in\left[0, \frac{t}{4}\right]\right) \\
& \quad \times P_{x_{\max }}\left(S_{\frac{t}{2}}=0\right) \\
& \geq \exp \left\{-t^{\mu+\epsilon}-\frac{t}{4}\right\}
\end{aligned}
$$

for sufficiently large $t$, which is the desired bound.

THE FIRST REGIME $\rho \in\left(\left(\frac{\alpha+1}{2 \alpha} 1_{d=1}+\frac{d}{2 \alpha} 1_{d \geq 2}\right) \vee 1, \frac{\alpha+d}{\alpha}\right]$ : In this case, the right hand side of (2.3) is sub-exponential and hence we must choose $\mu \leq 1$. We introduce a stopping time

$$
\zeta_{t}=\inf \left\{u \geq 0: \ell_{u}\left(x_{\max }\right) \geq \frac{t^{\rho}}{z\left(x_{\max }\right)}\right\}
$$

where $\ell$ denotes the occupation time of the random walk. Note that $S_{\zeta_{t}}=x_{\max }$ almost surely. Then, the strong Markov property and Lemma $\square$ show

$$
\begin{aligned}
& P_{0}\left(A_{t} \geq t^{\rho}, S_{t}=0\right) \\
& \quad \geq P_{0}\left(S_{\frac{t}{4}}=x_{\max }\right) E_{x_{\max }}\left[p_{\frac{3 t}{4}-\zeta_{t}}\left(x_{\max }, 0\right) 1_{\left\{\zeta_{t} \leq \frac{t}{4}\right\}}\right] \\
& \quad \geq \exp \left\{-t^{2 \mu-1+\epsilon}\right\} P_{0}\left(\ell_{\frac{t}{4}}(0) \geq t^{\rho-\frac{d \mu}{\alpha}+\epsilon}\right)
\end{aligned}
$$


for sufficiently large $t$. In order to bound the second factor, we employ the following result:

Theorem 6 (Theorem 1 in [12]). Suppose $f$ is a nonnegative bounded function with a finite support containing the origin and

$$
\lim _{t \rightarrow \infty} \sup _{x \in \operatorname{supp} f}\left|\frac{1}{a(t)} E_{x}\left[\int_{0}^{t} f\left(S_{u}\right) \mathrm{d} u\right]-1\right|=0 .
$$

Let $1 \ll b(t) \ll t$. Then for $\lambda>0$,

$$
\limsup _{t \rightarrow \infty} \frac{1}{b(t)} \log P_{0}\left(\int_{0}^{t} f\left(S_{u}\right) \mathrm{d} u \geq \lambda a\left(\frac{t}{b(t)}\right) b(t)\right) \leq-1-\log \frac{\lambda}{4}
$$

and for $\lambda \in(0,1)$,

$$
\liminf _{t \rightarrow \infty} \frac{1}{b(t)} \log P_{0}\left(\int_{0}^{t} f\left(S_{u}\right) \mathrm{d} u \geq \lambda a\left(\frac{t}{b(t)}\right) b(t)\right) \geq-\log \frac{1+\lambda}{1-\lambda} .
$$

Remark 6. An inspection of the proof in [12] shows that the upper bound holds even for functions $f_{t}$ depending on $t$ provided that it is non-negative and satisfies

$$
\limsup _{t \rightarrow \infty} \frac{1}{a\left(\frac{t}{b(t)}\right)} \sup _{x \in \operatorname{supp} f_{t}} E_{x}\left[\int_{0}^{\frac{t}{b(t)}} f_{t}\left(S_{u}\right) \mathrm{d} u\right] \leq 1 .
$$

This version will be used in the proof of the upper bound. See $\mathbb{A}$ for a proof.

We apply this to $f=1_{\{0\}}$ so that $\int_{0}^{t} f\left(S_{u}\right) \mathrm{d} u=\ell_{t}(0)$ and

$$
a(t)= \begin{cases}c \sqrt{t}, & d=1 \\ t^{o(1)}, & d \geq 2\end{cases}
$$

The choice

$$
b(t)= \begin{cases}t^{2 \rho-\frac{2 \mu}{\alpha}-1+2 \epsilon}, & d=1, \\ t^{\rho-\frac{d \mu}{\alpha}+2 \epsilon}, & d \geq 2\end{cases}
$$

makes

$$
a\left(\frac{t}{b(t)}\right) b(t)= \begin{cases}c \sqrt{t b(t)}=c t^{\rho-\frac{\mu}{\alpha}+\epsilon}, & d=1 \\ t^{o(1)} b(t)^{1+o(1)} \leq t^{\rho-\frac{d \mu}{\alpha}+\epsilon}, & d \geq 2\end{cases}
$$

for sufficiently large $t$ and hence we get, with $\lambda=\frac{1}{2}$ (say),

$$
P_{0}\left(\ell_{t}(0) \geq \frac{c}{2} t^{\rho-\frac{d \mu}{\alpha}+\epsilon}\right) \geq \begin{cases}\exp \left\{-c t^{2 \rho-\frac{2 \mu}{\alpha}-1+2 \epsilon}\right\}, & d=1, \\ \exp \left\{-c t^{\rho-\frac{d \mu}{\alpha}+2 \epsilon}\right\}, & d \geq 2,\end{cases}
$$


By doing the trivial change of variable $t \mapsto \frac{t}{4}$ and slightly changing $\epsilon$, we obtain

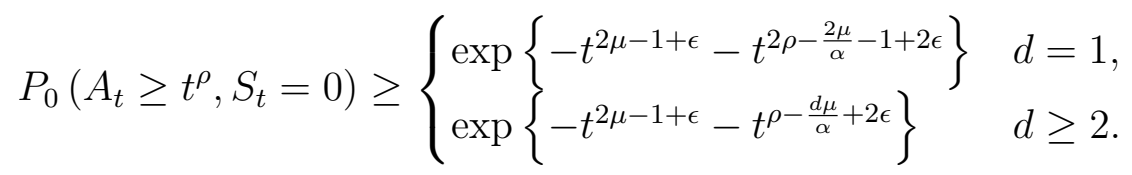

Optimizing over $\mu$, that is attained at

$$
\mu= \begin{cases}\frac{\alpha \rho}{\alpha+1}, & d=1 \\ \frac{\alpha(\rho+1)}{2 \alpha+d}, & d \geq 2\end{cases}
$$

which are in $\left(\frac{1}{2}, 1\right]$ in this regime, we find the desired lower bound.

THE THIRD REGIME $\rho<\left(\frac{\alpha+1}{2 \alpha} 1_{d=1}+\frac{d}{2 \alpha} 1_{d \geq 2}\right) \vee 1$ : We first give the proof for $d=1$. Let us start with the case $\alpha>1$ and $\rho<1$. In this case, $P_{0}\left(A_{\frac{t}{2}}>t^{\rho}\right)$ converges to one by the law of large numbers (ए.]). Thus for sufficiently large $M>0$, we have $P_{0}\left(A_{\frac{t}{2}}>t^{\rho}, S_{\frac{t}{2}}<M t^{\frac{1}{2}}\right) \geq \frac{1}{2}$. By using the Markov property and the local central limit theorem, we conclude that

$$
\begin{aligned}
& P_{0}\left(A_{t}>t^{\rho}, S_{t}=0\right) \\
& \quad \geq P_{0}\left(A_{\frac{t}{2}}>t^{\rho}, S_{\frac{t}{2}}<M t^{\frac{1}{2}}, S_{t}=0\right) \\
& \quad \geq t^{-\frac{1}{2}-\epsilon}
\end{aligned}
$$

for any $\epsilon>0$.

Next, for $\alpha \leq 1$ and $\rho<\frac{\alpha+1}{2 \alpha}$, we let the random walk go to the highest point of $z$-field in $\left[-t^{\frac{1}{2}}, t^{\frac{1}{2}}\right]$ within time $\frac{t}{4}$, leave the local time larger than $t^{\frac{1}{2}}$ there by the time $\frac{t}{2}$ and come back to the origin at $t$. Since the above highest value of $z$ is $t \frac{1}{2 \alpha}+o(1)$ $\mathbb{P}$-almost surely, we have $A_{t} \geq t^{\frac{1}{2}+\frac{1}{2 \alpha}+o(1)}$. On the other hand, the probability of this event is bounded from below by a power of $t$ by the local central limit theorem for $S_{t}$ and the central limit theorem for $\ell_{t}(0)$ in Darling-Kac [15].

In higher dimensions, if $\alpha>\frac{d}{2}$ and $\rho<1$ one can follow the same strategy as above. When $\alpha \leq \frac{d}{2}$ and $\rho<\frac{d}{2 \alpha}$, note that the highest point of $z$-field in $\left[-t^{\frac{1}{2}}, t^{\frac{1}{2}}\right]^{d}$ is $t^{\frac{d}{2 \alpha}+o(1)} \gg t^{\rho} \mathbb{P}$-almost surely. Thus it suffices to let the random walk stay there for the unit time instead of $t^{\frac{1}{2}}$. The rest of the argument is the same.

4.2. Upper bound of Theorem T: the second regime. In the second regime, that is, when $\rho>\frac{\alpha+d}{\alpha}$, the proof of the upper bound is very simple. We set

$$
\mu=\frac{\alpha(\rho-1)}{d}-\epsilon>1
$$

for small $\epsilon>0$ so that

$$
\max _{|x| \leq t^{\mu}} z(x)=t^{\frac{d \mu}{\alpha}+o(1)}<t^{\rho-1}
$$


as $t$ tends to infinity, $\mathbb{P}$-almost surely. This implies that $\left\{\max _{0 \leq u \leq t}\left|S_{u}\right| \leq t^{\mu}\right\} \cap\left\{A_{t} \geq\right.$ $\left.t^{\rho}\right\}=\emptyset$ (up to a $\mathbb{P}$-null set) for sufficiently large $t$. Therefore,

$$
\begin{aligned}
P_{0}\left(\int_{0}^{t} z\left(S_{u}\right) \mathrm{d} u \geq t^{\rho}\right) & \leq P_{0}\left(\max _{0 \leq u \leq t}\left|S_{u}\right|>t^{\mu}\right) \\
& \leq \exp \left\{-t^{\mu+o(1)}\right\},
\end{aligned}
$$

where we have used Lemma $\square$ and the reflection principle.

4.3. Upper bound of Theorem 17: the first regime. In the proof of the lower bound, we concentrate on the contribution from the highest peak of $z$-field. What remains to show is that the contributions from lower values of $z$ are negligible more precisely, it is much harder for the random walk to leave the right amount of occupation time on lower level sets of $z$. Throughout this subsection, we fix

$$
\mu= \begin{cases}\frac{\alpha \rho}{\alpha+1}, & d=1 \text { and } \rho \in\left[\frac{\alpha+1}{2 \alpha} \vee 1, \frac{\alpha+1}{\alpha}\right], \\ \frac{\alpha(\rho+1)}{2 \alpha+d}, & d \geq 2 \text { and } \rho \in\left[\frac{d}{2 \alpha} \vee 1, \frac{\alpha+d}{\alpha}\right],\end{cases}
$$

which are the optimal values found in the proofs of lower bounds (cf. (4.16)) . In view of (4.2), we may replace $z$ by

$$
\tilde{z}(x)=\left(z(x) \wedge t^{\frac{d \mu}{\alpha}+\epsilon}\right) 1_{\left\{|x| \leq t^{\mu}\right\}}
$$

for a small fixed $\epsilon>0$.

We define the level sets of $\tilde{z}$-field by $\mathcal{H}_{0}=\mathbb{Z}^{d}$ and

$$
\mathcal{H}_{k}=\mathcal{H}_{k}(t)=\left\{x \in \mathbb{Z}^{d}: \tilde{z}(x) \geq t^{k \epsilon}\right\} \quad \text { for } k \geq 1 \text {. }
$$

Note that $\mathcal{H}_{k}=\emptyset$ if $k>K:=\left[\frac{d \mu}{\epsilon \alpha}\right]$. Our starting point is the following obvious bound:

$$
\begin{aligned}
P_{0}\left(A_{t} \geq t^{\rho}\right) \leq & \sum_{k=0}^{K} P_{0}\left(\ell_{t}\left(\mathcal{H}_{k} \backslash \mathcal{H}_{k+1}\right) \geq \frac{1}{K+1} t^{\rho-(k+1) \epsilon}\right) \\
& +P_{0}\left(\max _{0 \leq u \leq t}\left|S_{u}\right|>t^{\mu}\right) .
\end{aligned}
$$

Thanks to Lemma 四, we have

$$
P_{0}\left(\max _{0 \leq u \leq t}\left|S_{u}\right|>t^{\mu}\right)=\exp \left\{-t^{2 \mu-1+o(1)}\right\}
$$

(recall $\mu \leq 1$ ) and hence the second term on the right hand side is harmless.

We bound each summand in the first term by applying the version of Theorem [ 6 mentioned in Remark [ to $f_{t}=1_{\mathcal{H}_{k}}$ (see also 因). The input required in the upper bound is the asymptotics of $a\left(\frac{t}{b_{t}}\right)$. (Note that when $f$ depends on $t$, we cannot relate this to that of $a(t)$.) Therefore we start by studying

$$
E_{x}\left[\int_{0}^{t^{\eta}} f\left(S_{u}\right) \mathrm{d} u\right]=E_{x}\left[\ell_{t^{\eta}}\left(\mathcal{H}_{k}\right)\right]
$$


for $\eta>0$.

Lemma 2. For any $\eta>0$ and $d \geq 1$, there exists a positive constant $c$ such that the $\mathbb{P}$-probability of

$$
\sup _{|x| \leq t^{\mu}} E_{x}\left[\ell_{t^{\eta}}\left(\mathcal{H}_{k}\right)\right] \leq c \begin{cases}t^{\frac{\eta}{2}}(\log t)^{3}, & d=1 \text { and } k \epsilon>\frac{\eta}{2 \alpha} \\ t^{\epsilon}, & d \geq 2 \text { and } k \epsilon>\frac{\eta}{\alpha}\end{cases}
$$

is bounded from below by $1-c \exp \left\{-c^{-1}(\log t)^{2}\right\}$. In particular, the above holds for all sufficiently large $t, \mathbb{P}$-almost surely.

Proof. Let us first explain the outline of the proof. It is straightforward to check that the mean $\mathbb{E} E_{x}\left[\ell_{t^{\eta}}\left(\mathcal{H}_{k}\right)\right]$ satisfies the desired bound. Then we show a concentration around the mean, which should not be hard since $E_{x}\left[\ell_{t^{\eta}}\left(\mathcal{H}_{k}\right)\right]$ is a linear functional of i.i.d. random variables $\left\{1_{\left\{\tilde{z}(y) \geq t^{k \epsilon}\right\}}\right\}_{y \in \mathbb{Z}^{d}}$. However, if we apply the martingale difference method directly to this family, then it turns out that we have too many terms. It is necessary (and natural) to take into account that these random variables degenerate as $t \rightarrow \infty$. To this end, we shall divide $\left[-t^{\mu}, t^{\mu}\right]^{d}$ into smaller cubes $\left\{I_{j}\right\}_{j \geq 1}$ in such a way that $\mathbb{P}\left(\# I_{j} \cap \mathcal{H}_{k} \geq 1\right)$ is bounded away from 0 and 1 . Then we use a martingale difference type method (McDiarmid's inequality, Theorem 6.5 in [9] ) to $\left\{I_{j} \cap \mathcal{H}_{k}\right\}_{j \geq 1}$. That $\# I_{j} \cap \mathcal{H}_{k}$ is unbounded causes a little problem but a simple truncation argument resolves it. This is the role of trimmed sets defined below.

Now we start the proof. We prove the lemma for the special case

$$
\mathbb{P}(z(x)>r)=r^{-\alpha} \wedge 1
$$

and indicate how to deal with the general case in the proof. For any $x \in \mathbb{Z}^{d}$,

$$
\begin{aligned}
\mathbb{E} E_{x}\left[\ell_{t^{\eta}}\left(\mathcal{H}_{k}\right)\right] & =\int_{0}^{t^{\eta}} \sum_{y \in \mathbb{Z}^{d}} p_{u}(x, y) \mathbb{P}\left(\tilde{z}(y) \geq t^{k \epsilon}\right) \mathrm{d} u \\
& \leq \int_{0}^{t^{\eta}} \sum_{y \in \mathbb{Z}^{d}} p_{u}(x, y) t^{-\alpha k \epsilon} \mathrm{d} u \\
& \leq c \begin{cases}t^{\frac{\eta}{2}}, & d=1, \\
t^{\epsilon}, & d \geq 2,\end{cases}
\end{aligned}
$$

where we have used the assumptions on $k \epsilon$.

Next we show a concentration of $E_{x}\left[\ell_{t^{\eta}}\left(\mathcal{H}_{k}\right)\right]$ around its mean. Let $I_{j}$ be the cube $t^{\frac{\alpha k \epsilon}{d}}\left(j+\left[0, \frac{1}{2}\right)^{d}\right)\left(j \in \frac{1}{2} \mathbb{Z}^{d}\right)$ and define a trimmed set $\underline{\mathcal{H}}_{k} \subset \mathcal{H}_{k}$ by keeping only at most $(\log t)^{2}$ points in each $I_{j}$. (The way how to choose these points does not matter 
in what follows.) It is simple to check that

$$
\begin{aligned}
\mathbb{P}\left(\underline{\mathcal{H}}_{k} \neq \mathcal{H}_{k}\right) & \leq \sum_{j \in \frac{1}{2} \mathbb{Z}^{d}} \mathbb{P}\left(\#\left(\mathcal{H}_{k} \cap I_{j}\right)>(\log t)^{2}\right) \\
& \leq 2^{-(\log t)^{2}} \#\left\{j \in \frac{1}{2} \mathbb{Z}^{d}: I_{j} \cap\left[-t^{\mu}, t^{\mu}\right]^{d} \neq \emptyset\right\} \\
& \leq 2^{-\frac{1}{2}(\log t)^{2}}
\end{aligned}
$$

by using the bound

$$
\begin{aligned}
\mathbb{P} & \left(\#\left(\mathcal{H}_{k} \cap I_{j}\right) \geq(\log t)^{2}\right) \\
& \leq\left(\begin{array}{c}
\left|I_{j}\right| \\
(\log t)^{2}
\end{array}\right) \mathbb{P}\left(z(x)>t^{k \epsilon}\right)^{(\log t)^{2}} \\
& \left.\leq\left(\left|I_{j}\right| t^{-\alpha k \epsilon}\right)\right)^{(\log t)^{2}} \\
& =2^{-d(\log t)^{2}} .
\end{aligned}
$$

Remark 7. In order to deal with a general distribution of the form ([2. $)$, we modify the side-length $L_{t}$ of $I_{j}$ as

$$
L_{t}^{d} \mathbb{P}\left(z(x)>t^{k \epsilon}\right) \sim \frac{1}{2} \text { as } t \rightarrow \infty .
$$

In the rest of the proof, we replace $t^{\frac{\alpha k \epsilon}{d}}$ by $L_{t}$ which is still $t^{\frac{\alpha k \epsilon}{d}+o(1)}$.

We are going to apply McDiarmid's inequality (Theorem 6.5 in [G] ) to $E_{x}\left[\ell_{t^{\eta}}\left(\underline{\mathcal{H}}_{k}\right)\right]$, regarding it as a function of independent random variables $\left\{I_{j} \cap \underline{\mathcal{H}}_{k}\right\}_{j \in \frac{1}{2} \mathbb{Z}^{d}}$. Let $x=0$ for simplicity and define $\mathbb{H}_{k}$ as the set of all possible realizations of $\underline{\mathcal{H}}_{k}$. Then the influence caused by changing the configuration in $I_{j}$ is bounded as

$$
\begin{aligned}
\Delta_{j} & =\sup _{\underline{\mathcal{H}}_{k}, \underline{\mathcal{H}}_{k}^{\prime} \in \mathbb{H}_{k}}\left|E_{0}\left[\ell_{t^{\eta}}\left(\underline{\mathcal{H}}_{k}\right)\right]-E_{0}\left[\ell_{t^{\eta}}\left(\underline{\mathcal{H}}_{k}^{\prime}\right)\right]\right| \\
& \leq \sup \left\{\int_{0}^{t^{\eta}} \sum_{y \in Y} p_{u}(0, y) \mathrm{d} u: Y \subset I_{j} \text { and } \# Y \leq(\log t)^{2}\right\} .
\end{aligned}
$$

Let us consider the case $d=1$ or 2 first. Lemma $\square$ implies

$$
p_{u}(0, y) \leq c\left(u^{-\frac{d}{2}} \wedge 1\right) \begin{cases}\exp \left\{-\frac{|y|^{2}}{c t^{\eta}}\right\}, & |y| \leq t^{\eta}, \\ c \exp \left\{-\frac{|y|}{c}\right\}, & |y|>t^{\eta}\end{cases}
$$

for $u \leq t^{\eta}$. For $|j| \leq 1$, we simply replace $y$ in (4.34) by 0 and obtain

$$
\begin{aligned}
\text { R.H.S. of }(4.33) & \leq(\log t)^{2} \int_{0}^{t^{\eta}} c\left(u^{-\frac{d}{2}} \wedge 1\right) \mathrm{d} u \\
& \leq c \begin{cases}t^{\frac{\eta}{2}}(\log t)^{2}, & d=1, \\
(\log t)^{3}, & d=2 .\end{cases}
\end{aligned}
$$


Next, for $1<|j| \leq t^{\eta-\frac{\alpha k \epsilon}{d}}$, since the worst case is to have all the points of $Y \subset I_{j}$ closest to the origin, we have

$$
\begin{aligned}
\text { R.H.S. of }(4.3 .3) & \leq(\log t)^{2} \int_{0}^{t^{\eta}} c\left(u^{-\frac{d}{2}} \wedge 1\right) \exp \left\{-\frac{t^{\frac{2 \alpha k \epsilon}{d}-\eta}(|j|-1)^{2}}{c}\right\} \mathrm{d} u \\
& \leq c \exp \left\{-\frac{t^{\frac{2 \alpha k \epsilon}{d}-\eta}(|j|-1)^{2}}{c}\right\} \begin{cases}t^{\frac{\eta}{2}}(\log t)^{2}, & d=1, \\
(\log t)^{3}, & d=2\end{cases}
\end{aligned}
$$

by using the first line of (4.34). Finally for $|j|>t^{\eta-\frac{\alpha k \epsilon}{d}} \vee 1$, one can see that

$$
\text { R.H.S. of }(4.3: 3) \leq \exp \{-c|j|\}
$$

again by considering the worst case and using the second line of (4.34). The sum of this part is always bounded.

Now we can complete the case $d=1$. For $k \epsilon>\frac{\eta}{2 \alpha}$, the off-diagonal part (4.36) is stretched exponentially small and

$$
\begin{aligned}
\sum_{j} \Delta_{j}^{2} & \leq \sum_{|j| \leq 1}\left[\text { R.H.S. of }\left(\frac{4.3 .5)}{2}\right]^{2}+\sum_{j \geq 2}[\text { R.H.S. of }(4.36)]^{2}+O(1)\right. \\
& =c t^{\eta}(\log t)^{4}+O(1) .
\end{aligned}
$$

Therefore, McDiarmid's inequality implies

$$
\begin{aligned}
& \mathbb{P}\left(\left|E_{0}\left[\ell_{t^{\eta}}\left(\underline{\mathcal{H}}_{k}\right)\right]-\mathbb{E} E_{0}\left[\ell_{t^{\eta}}\left(\underline{\mathcal{H}}_{k}\right)\right]\right|>t^{\frac{\eta}{2}}(\log t)^{3}\right) \\
& \quad \leq c \exp \left\{-\frac{t^{\eta}(\log t)^{6}}{c \sum_{j} \Delta_{j}^{2}}\right\} \\
& \quad=c \exp \left\{-\frac{1}{2 c}(\log t)^{2}\right\} .
\end{aligned}
$$

Using (4.2.9) and the fact that $\# \mathcal{H}_{k}$ is at most polynomial in $t$ (recall that $\mathcal{H}_{k}$ are subsets of $\left[-t^{\mu}, t^{\mu}\right]$ by the definition of $\left.\tilde{z}\right)$, we are done.

The argument for the case $d=2$ is very similar to $d=1$. For $k \epsilon>\frac{\eta}{\alpha}$, the part (4.36) is again stretched exponentially small and hence

$$
\begin{aligned}
\sum_{j} \Delta_{j}^{2} & \leq \sum_{|j| \leq 1}\left[\text { R.H.S. of }\left(\frac{4.35)}{2}\right]^{2}+\sum_{j \geq 2}[\text { R.H.S. of }(4.36)]^{2}+O(1)\right. \\
& =c(\log t)^{6}+O(1) .
\end{aligned}
$$

This bounds and McDiarmid's inequality yield

$$
\mathbb{P}\left(\left|E_{0}\left[\ell_{t}\left(\underline{\mathcal{H}}_{k}\right)\right]-\mathbb{E} E_{0}\left[\ell_{t}\left(\underline{\mathcal{H}}_{k}\right)\right]\right|>(\log t)^{4}\right) \leq c \exp \left\{-\frac{1}{2 c}(\log t)^{2}\right\}
$$

and the rest is the same as before. 
Finally we settle the case $d \geq 3$. This case is simpler since we know

$$
\int_{0}^{t^{\eta}} p_{u}(0, y) \mathrm{d} u \leq \int_{0}^{\infty} p_{u}(0, y) \mathrm{d} u \leq c(|y|+1)^{2-d} .
$$

Using this in (4.3:3), we obtain

$$
\begin{aligned}
\sum_{j} \Delta_{j}^{2} & \leq(\log t)^{4} \sum_{j} \sup _{y \in I_{j}}\left(\int_{0}^{t^{\eta}} p_{u}(0, y) \mathrm{d} u\right)^{2} \\
& \leq c(\log t)^{4} \sum_{j}\left(t^{\frac{\alpha k \epsilon}{d}}|j|+1\right)^{4-2 d} .
\end{aligned}
$$

This is always $O\left(\left(\log t^{4}\right)\right)$ and the rest is routine.

The second part of the lemma follows from the first one and the Borel-Cantelli lemma.

We use this lemma, together with Theorem [6, to bound

$$
P_{0}\left(\ell_{t}\left(\mathcal{H}_{k} \backslash \mathcal{H}_{k+1}\right)>\frac{1}{K+1} t^{\rho-(k+1) \epsilon}\right) \leq P_{0}\left(\ell_{t}\left(\mathcal{H}_{k}\right)>\frac{1}{K+1} t^{\rho-(k+1) \epsilon}\right)
$$

Note first that this probability is zero if $k<\frac{\rho-1}{\epsilon}-1$ as the total mass of $\ell_{t}$ is $t$. Hence we are only concerned with

$$
\rho-1-\epsilon \leq k \epsilon \leq \frac{d \mu}{\alpha}+\epsilon
$$

Let us start with the case $d=1$. We choose $b(t)=t^{2 \rho-1-2(k+2) \epsilon}$ and $\eta=2(1+(k+$ $2) \epsilon-\rho$ ). Thanks to (4.45), one can verify that $k \epsilon>\frac{\eta}{2 \alpha}$ for sufficiently small $\epsilon$. This allows us to use Lemma $\nabla$ to obtain

$$
4 a\left(\frac{t}{b(t)}\right) b(t)<\frac{1}{K+1} t^{\rho-(k+1) \epsilon} .
$$

Therefore, Theorem [ 6 yields the bound

$$
\begin{aligned}
P_{0} & \left(\ell_{t}\left(\mathcal{H}_{k} \backslash \mathcal{H}_{k+1}\right)>\frac{1}{K+1} t^{\rho-(k+1) \epsilon}\right) \\
& \leq P_{0}\left(\ell_{t}\left(\mathcal{H}_{k} \backslash \mathcal{H}_{k+1}\right)>4 a\left(\frac{t}{b(t)}\right) b(t)\right) \\
& \leq \exp \{-b(t)\} \\
& =\exp \left\{-c t^{2 \rho-1-2(k+2) \epsilon}\right\} .
\end{aligned}
$$

Since this is increasing in $k$, the largest upper bound is for $k=K$ and then $b(t)=t^{p(\alpha, \rho)-c \epsilon}$. Now all the terms on the right hand side of $(4.24)$ are bounded by $\exp \left\{-t^{p(\alpha, \rho)-c \epsilon}\right\}$ and the proof is completed. 
The case $d \geq 2$ is almost the same. We choose $\eta=1-\rho+(k+3) \epsilon$ and $b(t)=$ $t^{\rho-(k+3) \epsilon}$. One can check that this satisfies $k \epsilon>\frac{\eta}{\alpha}$ with the help of (4.27) and hence by Lemma 2 ,

$$
4 a\left(\frac{t}{b(t)}\right) b(t)<\frac{1}{K+1} t^{\rho-(k+1) \epsilon} .
$$

Then, just as above, we obtain

$$
P_{0}\left(\ell_{t}\left(\mathcal{H}_{k} \backslash \mathcal{H}_{k+1}\right)>\frac{1}{K+1} t^{\rho-(k+1) \epsilon}\right)=\exp \left\{-c t^{\rho-(k+3) \epsilon}\right\}
$$

which is largest and matches (2.3) at $k=K$.

Remark 8. Let us comment on the technical difference between our proof and the earlier works [3, 2, 10] that contain quenched tail estimates. We focus on the upper bound which is more involved in the present model.

The arguments in [3, 2, [10] rely on the spectral theoretic technics which were developed in the study of the so-called parabolic Anderson model. More precisely, they obtain the upper bound through the exponential moment (with a small parameter $\left.\epsilon_{t}\right)$ of $A_{t}$, using the fact that it is a solution of $\partial_{t} u=\left(\Delta+\epsilon_{t} z\right) u$. By restricting the problem to a certain macro-box as we have done above and using the eigenfunction expansion, the problem is reduced to the behavior of the largest eigenvalue of $\Delta+\epsilon_{t} z$ in a large box. Then a localization procedure demonstrated in [22] (Proposition 1) allows them to bound it by the maximum of eigenvalues among smaller sub-boxes. A crucial ingredient to control the maximum of local eigenvalue is a large deviation principle for a scaled $z$-field in a suitable function space, Lemma 2.1 in [2] or Theorem 1 in [10].

In our heavy tailed setting, it seems difficult to find a substitute for the large deviation principle for the $z$-field. The above proof reveals that the relevant part of the $z$-field looks like a delta function. This makes the choice of function space (or topology) a delicate problem.

4.4. Proof of Theorem 2. We follow the same strategy as in the previous subsection. Fix $c^{\prime} \in(\mathbb{E}[z(0)], c)$ and bound the large deviation probability as

$$
\begin{aligned}
P_{0}\left(A_{t} \geq c t\right) \leq & P_{0}\left(\int_{0}^{t} z\left(S_{u}\right) \wedge t^{l \epsilon} \mathrm{d} u \geq c^{\prime} t\right) \\
& +\sum_{k=l}^{K} P_{0}\left(\ell_{t}\left(\mathcal{H}_{k} \backslash \mathcal{H}_{k+1}\right) \geq \frac{c-c^{\prime}}{K+1} t^{1-(k+1) \epsilon}\right) \\
& +P_{0}\left(\max _{0 \leq u \leq t}\left|S_{u}\right|>t^{\mu}\right),
\end{aligned}
$$

where we set $\rho=1$ in the definition of $K$ and $\mu$. The third term is negligible as before. As for the second term, we use the same level sets $\mathcal{H}_{k}$ and $\eta$ as in the previous subsection. Then with $\rho=1$, we see that the assumptions of Lemma $\nabla$ hold 
for $k>\frac{2}{\alpha-1}(d=1)$ and $k>\frac{3}{\alpha-1}(d \geq 2)$ and hence (4.47) and (4.4.9) respectively. Thus if we set $l$ to be the smallest integer larger than $\frac{2}{\alpha-1}(d=1)$ and $\frac{3}{\alpha-1}(d \geq 2)$, then the summand with $k=K$ dominates all the others. Since it has the desired asymptotics, it remains to show that the first term in (4.50) is negligible.

In order to simplify the notation, we rename $l \epsilon$ to $\epsilon$ and $c^{\prime}$ to $c$. We are going to show that for any $\epsilon \in\left(0, \frac{1}{20}\right), \mathbb{P}$-almost surely,

$$
P_{0}\left(\int_{0}^{t} z\left(S_{u}\right) \wedge t^{\epsilon} \mathrm{d} u>c t\right) \leq \exp \left\{-t^{1-7 \epsilon}\right\}
$$

for large $t$. This proves Theorem $\square$ since the exponents in (¿.8) are strictly less than one. Our argument is again based on [1:2] (the proof of Theorem 2 there, presented in Section 3) and hence it is natural to begin with the following type of bound.

Lemma 3. Let $\epsilon>0$. Then $\mathbb{P}$-almost surely, for all sufficiently large $t$,

$$
\sup _{|x| \leq t}\left|E_{x}\left[\int_{0}^{T} z\left(S_{u}\right) \wedge t^{\epsilon} \mathrm{d} u\right]-T \mathbb{E}[z(0)]\right| \leq \frac{T}{\log t}
$$

holds for any $T \in\left[t^{5 \epsilon}, t\right]$.

Proof. One can check that it suffices to prove the claim for $z 1_{[-2 t, 2 t]^{d}}$ instead of $z$. Henceforth, every configuration appearing below is set to be zero outside $[-2 t, 2 t]^{d}$. The proof is similar to that of Lemma 20. The average over the random scenery is close to $T \mathbb{E}[z(0)]$. Indeed, there exists $c>0$ such that

$$
\begin{aligned}
\left|\mathbb{E} E_{x}\left[\int_{0}^{T} z\left(S_{u}\right) \wedge t^{\epsilon} \mathrm{d} u\right]-T \mathbb{E}[z(0)]\right| & =T \mathbb{E}\left[\left(z(0)-t^{\epsilon}\right)_{+}\right] \\
& \leq \frac{c T}{t^{\epsilon(\alpha-1) / 2}}
\end{aligned}
$$

for any $t>0$, uniformly in $T \in\left[t^{\epsilon}, t\right]$ and $x \in[-t, t]^{d}$. Note that this is much smaller than $T / \log t$ for sufficiently large $t>0$ since we are considering $\alpha>1$. We shall bound the variation of $E_{x}\left[\int_{0}^{T} z\left(S_{u}\right) \wedge t^{\epsilon} \mathrm{d} u\right]$ as a functional of $z$. For two configurations $z_{1}$ and $z_{2}$, by using the Cauchy-Schwarz inequality and the Chapman-Kolmogorov 
identity, we have

$$
\begin{aligned}
& \left|E_{x}\left[\int_{0}^{T} z_{1}\left(S_{u}\right) \mathrm{d} u\right]-E_{x}\left[\int_{0}^{T} z_{2}\left(S_{u}\right) \mathrm{d} u\right]\right| \\
& \quad=\left|\sum_{y \in[-2 t, 2 t]^{d}} \int_{0}^{T} p_{u}(x, y) z_{1}(y) \mathrm{d} u-\sum_{y \in[-2 t, 2 t]^{d}} \int_{0}^{T} p_{u}(x, y) z_{2}(y) \mathrm{d} u\right| \\
& \quad \leq \int_{0}^{T}\left(\sum_{y \in[-2 t, 2 t]^{d}} p_{u}(x, y)^{2}\right)^{\frac{1}{2}}\left(\sum_{y \in[-2 t, 2 t]^{d}}\left|z_{1}(y)-z_{2}(y)\right|^{2}\right)^{\frac{1}{2}} \mathrm{~d} u \\
& \quad \leq\left|z_{1}-z_{2}\right|_{2} \int_{0}^{T} p_{2 u}(x, x)^{\frac{1}{2}} \mathrm{~d} u \\
& \quad \leq c T^{\frac{3}{4}}\left|z_{1}-z_{2}\right|_{2} .
\end{aligned}
$$

This allows us to use the Talagrand's concentration inequality (Theorem 6.6 in [32] and the argument below (4.2) in the same paper to replace the median by the mean) to obtain the bound

$$
\begin{aligned}
& \mathbb{P}\left(\left|E_{x}\left[\int_{0}^{T} z\left(S_{u}\right) \wedge t^{\epsilon} \mathrm{d} u\right]-\mathbb{E} E_{x}\left[\int_{0}^{T} z\left(S_{u}\right) \wedge t^{\epsilon} \mathrm{d} u\right]\right| \geq \frac{T}{4 \log t}\right) \\
& \quad \leq c_{1} \exp \left\{-c_{2} \frac{T^{2}}{T^{\frac{3}{2}} t^{2 \epsilon}(\log t)^{2}}\right\} .
\end{aligned}
$$

One can check that the argument of the exponential is a positive power of $t$ for $T>t^{5 \epsilon}$ and $\epsilon<\frac{1}{20}$ From this and (4.53), we deduce the discretized bound

$$
\sup _{|x| \leq t}\left|E_{x}\left[\int_{0}^{T} z\left(S_{u}\right) \wedge t^{\epsilon} \mathrm{d} u\right]-T \mathbb{E}[z(0)]\right| \leq \frac{T}{4 \log t}
$$

uniformly in $T \in\left[t^{5 \epsilon}, t\right] \cap \mathbb{N}$ for all sufficiently large $t \in \mathbb{N}, \mathbb{P}$-almost surely, by the Borel-Cantelli lemma. This can be extended to continuum $T$, with a price of extra factor 2 on the right hand side, by a simple monotonicity argument. Moreover, as we vary $t$ over an interval $[\lfloor t\rfloor,\lfloor t\rfloor+1]$, the $\sup _{|x| \leq t}$ is monotone and the above integral varies at most $O\left(T t^{\epsilon-1}\right)$. Hence the above bound extends to continuum $t$ with another extra factor 2.

Now we repeat the argument in the proof of Theorem 2 in [12]. We write $\lambda=t^{-6 \epsilon}$ and $f(x)=z(x) \wedge t^{\epsilon}$ to simplify the notation. Let $P_{u} f(x)=E_{x}\left[f\left(S_{u}\right)\right]$ and

$$
u_{\lambda}(x)=\int_{0}^{\infty} e^{-\lambda u} P_{u} f(x) \mathrm{d} u=(\lambda-\mathcal{A})^{-1} f(x),
$$

where $\mathcal{A}=(2 d)^{-1} \Delta$ denotes the generator of the continuous time simple random walk. Note that $\mathbb{P}$-almost surely, $f$ is not identically zero and hence $u_{\lambda}(x)>0$ for 
all $x \in \mathbb{Z}^{d}$. By definition we have

$$
-\mathcal{A} u_{\lambda}(x)=u_{\lambda}(x)\left(v_{\lambda}(x)-\lambda\right)
$$

with $v_{\lambda}=f u_{\lambda}{ }^{-1}$, that is, $u_{\lambda}$ is harmonic for $\mathcal{A}+\left(v_{\lambda}-\lambda\right)$ and consequently

$$
u_{\lambda}(x)=e^{-\lambda t} E_{x}\left[u_{\lambda}\left(S_{t}\right) \exp \left\{\int_{0}^{t} v_{\lambda}\left(S_{u}\right) \mathrm{d} u\right\}\right] .
$$

By using Lemma 3 and the obvious bound $\int_{0}^{T} P_{u} f(x) \mathrm{d} u \leq T t^{\epsilon}$ for $T \geq 0$, we obtain

$$
u_{\lambda}(x) \sim \frac{\mathbb{E}[z(0)]}{\lambda}
$$

as $\lambda=t^{-6 \epsilon} \downarrow 0$ uniformly in $|x| \leq t$. Indeed, integration by parts shows

$$
\begin{aligned}
u_{\lambda}(x) & =\left[e^{-\lambda u} \int_{0}^{u} P_{s} f(x) \mathrm{d} s\right]_{u=0}^{\infty}+\int_{0}^{\infty} \lambda e^{-\lambda u} \int_{0}^{u} P_{s} f(x) \mathrm{d} s \mathrm{~d} u \\
& =\int_{t^{5 \epsilon}}^{t} \lambda e^{-\lambda u} u \mathbb{E}[z(0)] \mathrm{d} u+R(\lambda),
\end{aligned}
$$

where the first term is asymptotic to $\lambda^{-1} \mathbb{E}[z(0)]$ and $R(\lambda)$ is the remainder term. The latter is bounded as

$$
\begin{aligned}
|R(\lambda)| & \leq\left(\int_{0}^{t^{5 \epsilon}}+\int_{t}^{\infty}\right) \lambda e^{-\lambda u} t^{\epsilon} u \mathrm{~d} u+\frac{1}{\log t} \int_{t^{5 \epsilon}}^{t} \lambda e^{-\lambda u} u \mathrm{~d} u \\
& =o\left(\lambda^{-1}\right) .
\end{aligned}
$$

Using this in (4.59), we find

$$
\begin{aligned}
E_{0} & {\left[\exp \left\{\frac{\lambda}{\mathbb{E}[z(0)]+\epsilon} \int_{0}^{t} f\left(S_{u}\right) \mathrm{d} u\right\}: \max _{0 \leq u \leq t}\left|S_{u}\right| \leq t\right] } \\
& \leq E_{0}\left[\exp \left\{\int_{0}^{t} v_{\lambda}\left(S_{u}\right) \mathrm{d} u\right\}: \max _{0 \leq u \leq t}\left|S_{u}\right| \leq t\right] \\
& \leq \frac{1}{\min _{|x| \leq t} u_{\lambda}(x)} E_{0}\left[u_{\lambda}\left(S_{t}\right) \exp \left\{\int_{0}^{t} v_{\lambda}\left(S_{u}\right) \mathrm{d} u\right\}\right] \\
& \stackrel{(4.5 \mathrm{~g})}{\leq} e^{\lambda t} \frac{u_{\lambda}(0)}{\min _{|x| \leq t} u_{\lambda}(x)} \\
& \stackrel{(4.6 \mathrm{~d})}{=} e^{\lambda t}(1+o(1))
\end{aligned}
$$


as $t$ tends to infinity. Applying the Markov inequality, we arrive at

$$
\begin{aligned}
P_{0} & \left(\int_{0}^{t} f\left(S_{u}\right) \mathrm{d} u \geq c t, \max _{0 \leq u \leq t}\left|S_{u}\right| \leq t\right) \\
& \leq 2 \exp \left\{\lambda t-\frac{c t}{\mathbb{E}[z(0)] \lambda^{-1}}\right\} \\
& =2 \exp \left\{-\left(\frac{c}{\mathbb{E}[z(0)]}-1\right) t^{1-6 \epsilon}\right\} .
\end{aligned}
$$

As $P_{0}\left(\max _{0 \leq u \leq t}\left|S_{u}\right|>t\right)$ decays exponentially in $t$, this completes the proof of (4.5]).

\section{PROOFS FOR THE RANDOM WALK IN RANDOM LAYERED CONDUCTANCE}

5.1. Proofs of Theorems $\mathbf{3}$ and 4 . Recall that we write $x \in \mathbb{Z}^{1+d}$ as $\left(x_{1}, x_{2}\right)$ with $x_{1} \in \mathbb{Z}$ and $x_{2} \in \mathbb{Z}^{d}$. Also whenever one finds a point in $\mathbb{R}^{k}$ below, it should be understood as a closest lattice point.

Proof of Theorem . We give the proof only for $d=1$ since the higher dimensional case is almost the same. Without loss of generality we may assume $\mathbf{e}=\mathbf{e}_{2}$. Let $S^{1}$ and $S^{2}$ be continuous time simple random walks on $\mathbb{Z}$ independent of each other (strictly speaking, they have jump rates $\frac{1}{2}$ ). Then our process has the representation

$$
\left(X_{t}^{1}, X_{t}^{2}\right)_{t \geq 0}=\left(S_{A_{t}^{2}}^{1}, S_{t}^{2}\right)_{t \geq 0},
$$

where the clock process is defined by $A_{t}^{2}=\int_{0}^{t} z\left(S_{u}^{2}\right) \mathrm{d} u$. This representation allows us to write

$$
\begin{aligned}
P_{0}^{\omega}\left(X_{t}=t^{\delta} \mathbf{e}_{1}+t^{\gamma} \mathbf{e}_{2}\right) & =P_{0}^{\otimes 2}\left(S_{A_{t}^{2}}^{1}=t^{\delta}, S_{t}^{2}=t^{\gamma}\right) \\
& =E_{0}\left[p_{A_{t}^{2}}\left(0, t^{\delta}\right) 1_{\left\{S_{t}^{2}=t^{\gamma}\right\}}\right]
\end{aligned}
$$

The last formula involves only the second random walk $S^{2}$ and henceforth we drop the superscript.

UPPER BOUND: We first use Hölder's inequality to obtain

$$
\text { R.H.S. of }(5.2) \leq E_{0}\left[p_{A_{t}}\left(0, t^{\delta}\right)^{2}\right]^{\frac{1}{2}} P_{0}\left(S_{t}=t^{\gamma}\right)^{\frac{1}{2}}
$$

By Lemma 四, we have

$$
P_{0}\left(S_{t}=t^{\gamma}\right)=\exp \left\{-t^{(2 \gamma-1) \wedge \gamma+o(1)}\right\}
$$


as $t$ tends to infinity. On the other hand,

$$
\begin{aligned}
E_{0}\left[p_{A_{t}}\left(0, t^{\delta}\right)^{2}\right] \leq & E_{0}\left[\exp \left\{-\frac{c t^{2 \delta}}{A_{t}}\right\} 1_{\left\{A_{t} \geq t^{\delta}\right\}}\right]+E_{0}\left[\exp \left\{-c t^{\delta}\right\} 1_{\left\{A_{t}<t^{\delta}\right\}}\right] \\
\leq & \sum_{0 \leq k<t^{M}} \exp \left\{-\frac{c t^{2 \delta}}{t^{\delta}+k+1}\right\} P_{0}\left(t^{\delta}+k \leq A_{t}<t^{\delta}+k+1\right) \\
& +P_{0}\left(A_{t} \geq t^{M}\right)+E_{0}\left[\exp \left\{-c t^{\delta}\right\} 1_{\left\{A_{t}<t^{\delta}\right\}}\right] .
\end{aligned}
$$

The second term can be made negligible by choosing $M>0$ sufficiently large and using Theorem $\square$. The first term is bounded by

$$
t^{M} \sup _{\rho \in[\delta, M]} \exp \left\{-c t^{2 \delta-\rho}\right\} P_{0}\left(A_{t} \geq t^{\rho}\right)
$$

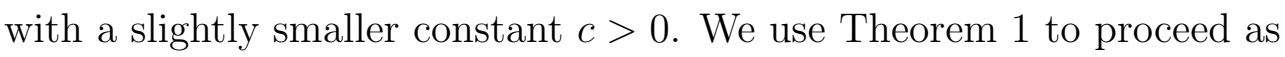

$$
\exp \left\{-c t^{2 \delta-\rho}\right\} P_{0}\left(A_{t} \geq t^{\rho}\right) \leq \exp \left\{-c t^{2 \delta-\rho}-t^{p(\alpha, \rho)-\epsilon}\right\}
$$

for any $\epsilon>0$, where $p(\alpha, \rho)$ is set to be zero for $\rho \leq \frac{\alpha+1}{2 \alpha} \vee 1$. Therefore, we arrive at an upper bound of the form

$$
E_{0}\left[p_{A_{t}}\left(0, t^{\delta}\right)^{2}\right] \leq \exp \left\{-c t^{q(\alpha, \delta)-\epsilon}\right\}
$$

with

$$
q(\alpha, \delta)=\delta \wedge\left(\inf _{\rho \in[\delta, M]} p(\alpha, \rho) \vee(2 \delta-\rho)\right)
$$

It is a simple matter of checking that this coincides with (2.]6).

Remark 9. We give a brief guide on the final step above for $d=1$. In what follows, the constant $M>0$ is chosen sufficiently large. Figures $\mathbb{\theta}$ and 1 might also help. Note that $p(\alpha, \rho)$ is increasing in $\rho$ whereas $2 \delta-\rho$ is decreasing. Hence the infimum over $\rho \in(0, \infty)$ of $p(\alpha, \rho) \vee(2 \delta-\rho)$ takes place at the point where $p(\alpha, \rho)=2 \delta-\rho$, which has a unique solution except for the case $\alpha>1$ and $\frac{1}{2}<\delta<\frac{\alpha}{\alpha+1}$. One can check that the minimizer lies outside $[\delta, M]$ if and only if $\alpha>1$ and $\delta>\frac{\alpha}{\alpha-1}$, in which case the infimum over $\rho \in[\delta, M]$ is attained at $\delta$. Based on these observations, one finds that

$$
\begin{cases}0, & \delta \leq \frac{1}{2} \vee \frac{\alpha+1}{4 \alpha} \\ \frac{4 \alpha \delta-\alpha-1}{3 \alpha+1}, & \frac{\alpha}{\alpha+1} \vee \frac{1}{2} \leq \delta<\frac{2 \alpha+1}{2 \alpha} \\ \frac{\alpha(2 \delta-1)}{\alpha+1}, & \frac{2 \alpha+1}{2 \alpha} \leq \delta<\frac{\alpha}{(\alpha-1)_{+}} \\ \frac{\alpha(\delta-1)}{d}, & \delta>\frac{\alpha}{(\alpha-1)_{+}}\end{cases}
$$




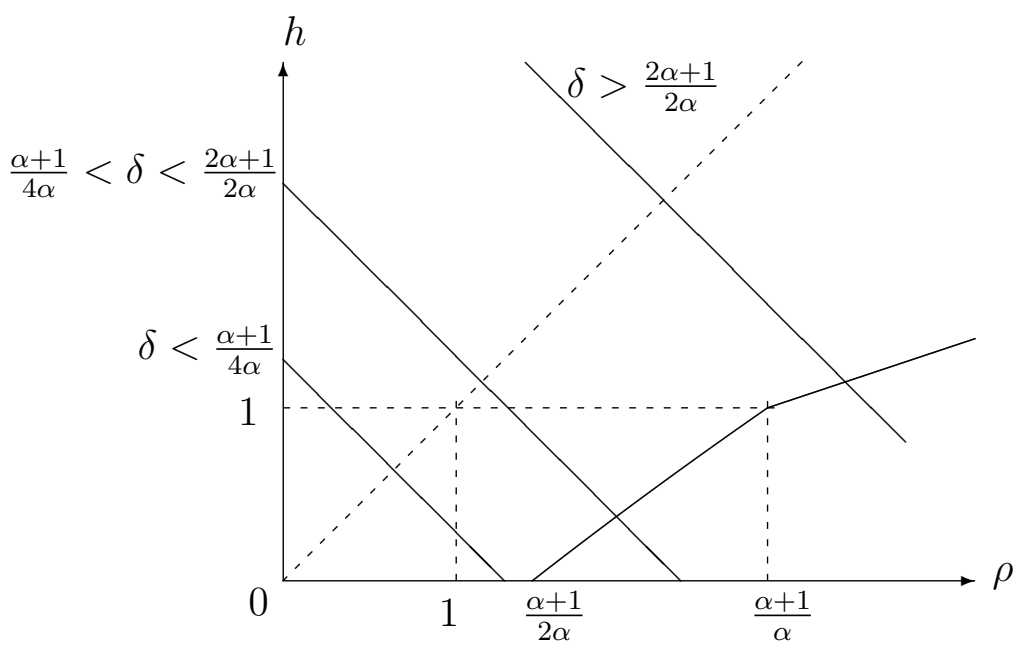

Figure 4 . The case $\alpha \leq 1$ : The decreasing lines are $h=2 \delta-\rho$. The increasing dashed line is $h=\rho$. The increasing piecewise linear curve, including the flat piece from $(0,0)$ to $\left(\frac{\alpha+1}{2 \alpha}, 0\right)$, is $h=p(\alpha, \rho)$. In this case, the slopes of the latter never exceed one. It follows that the point $2 \delta-\rho=p(\alpha, \rho)$ is always below $\delta$, that is, the point of $\rho=2 \delta-\rho$.

In the first three cases, the values are smaller than $\delta$ and the fourth one is larger than $\delta$. Finally, in the remaining case $\alpha>1$ and $\frac{1}{2}<\delta<\frac{\alpha}{\alpha+1}$, we have

$$
p(\alpha, \rho) \vee(2 \delta-\rho)= \begin{cases}2 \delta-\rho, & \rho \leq 1, \\ p(\alpha, \rho), & \rho>1\end{cases}
$$

and this takes the minimum value $2 \delta-1$ at $\rho=1 \in[\delta, M]$.

LOWER BOUND: Since the lower bounds in the first regimes in (2.16]) and (2.T7)

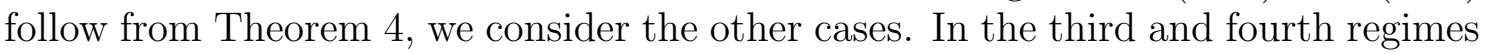
$\frac{\alpha+1}{4 \alpha} \vee \frac{\alpha}{\alpha+1}<\delta<\frac{\alpha}{(\alpha-1)_{+}}$, we set

$$
\rho= \begin{cases}\frac{(2 \delta+1)(\alpha+1)}{3 \alpha+1}<\frac{\alpha+1}{\alpha}, & \frac{\alpha+1}{4 \alpha} \vee \frac{\alpha}{\alpha+1}<\delta<\frac{2 \alpha+1}{2 \alpha}, \\ \frac{2 \delta+\alpha}{\alpha+1} \geq \frac{\alpha+1}{\alpha}, & \delta \geq \frac{2 \alpha+1}{2 \alpha}\end{cases}
$$

which gives the infimum in the proof of the upper bound. One can verify that this is larger than $\delta \vee 1 \vee \frac{\alpha+1}{2 \alpha}$ and hence $p(\alpha, \rho)$ is to be computed by (2.4). (This can be seen from Figures $\mathbb{G}$ and 5 . Indeed, the point where $p(\alpha, \delta)$ crosses $2 \delta-\rho$ lies in $\left\{\rho>1 \vee \frac{\alpha+1}{2 \alpha}\right\}$ and also on the right of the dashed line with slope 1.) Now we use the 


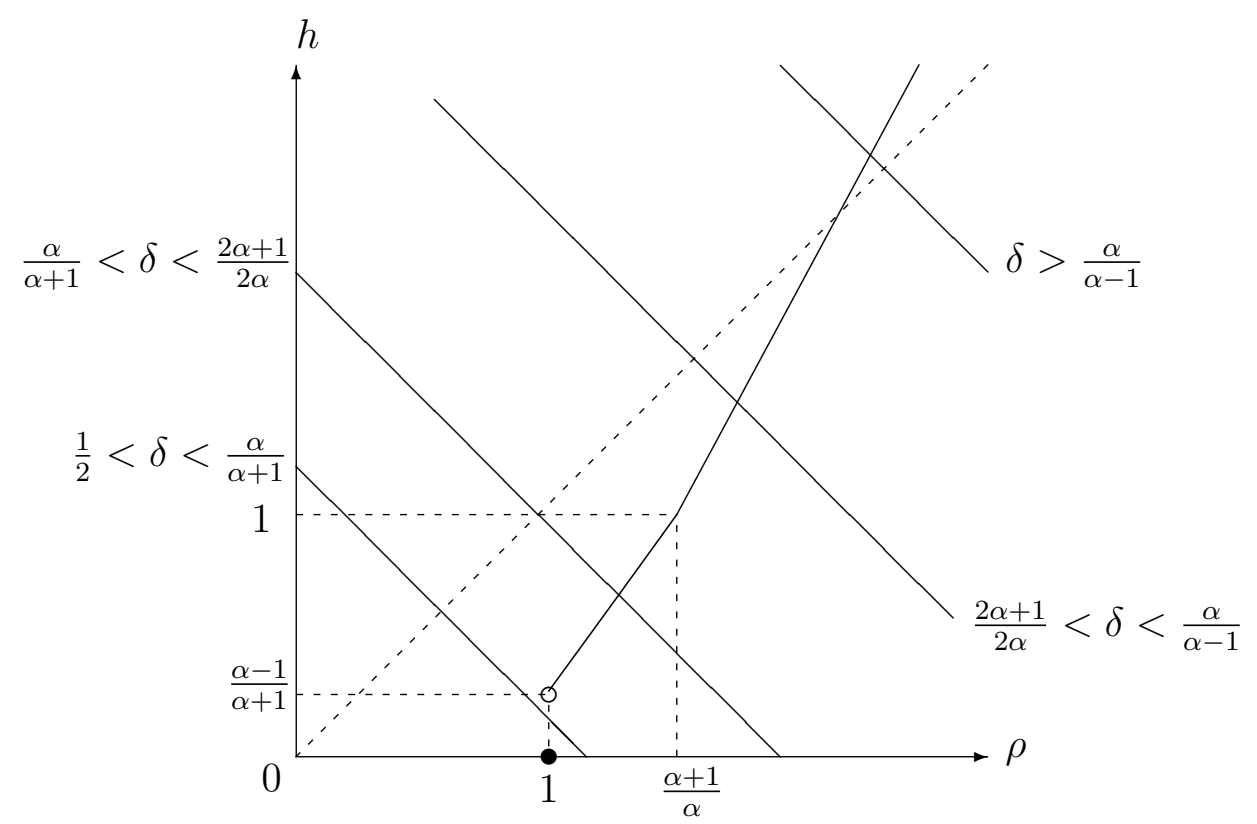

Figure 5. The case $\alpha>1$ : The decreasing lines are $h=2 \delta-\rho$. The increasing dashed line is $h=\rho$. The increasing piecewise linear curve, including the flat piece from $(0,0)$ to $(1,0)$, is $h=p(\alpha, \rho)$. When $\alpha>2$, the slope of the last piece exceeds one. Then the point of $2 \delta-\rho=p(\alpha, \rho)$ may be above $\delta$ that is, the point of $\rho=2 \delta-\rho$.

first part of Lemma $\square$ and the Markov property to obtain

$$
\begin{aligned}
& \text { R.H.S. of (5.2) } \\
& \begin{array}{l}
\geq E_{0}\left[p_{A_{t}}\left(0, t^{\delta}\right): S_{t}=t^{\gamma},\left(\frac{t}{2}\right)^{\rho} \leq A_{t} \leq t^{M}, S_{\frac{t}{2}}=0\right] \\
\geq \exp \left\{-t^{2 \delta-\rho+o(1)}\right\} P_{0}\left(\left(\frac{t}{2}\right)^{\rho} \leq A_{\frac{t}{2}}, S_{\frac{t}{2}}=0\right) P_{0}\left(S_{\frac{t}{2}}=t^{\gamma}\right) \\
\quad-P_{0}\left(A_{t}>t^{M}\right) .
\end{array}
\end{aligned}
$$

Theorem $\square$ shows that the last term in (5.13) decays faster than the desired lower bound for sufficiently large $M$. We use (4.3) for the second factor and Lemma $\square$ for the third factor to bound the first term from below by

$$
\exp \left\{-t^{(2 \delta-\rho) \vee p(\alpha, \rho) \vee((2 \gamma-1) \wedge \gamma)+o(1)}\right\} \text {. }
$$

This is the desired lower bound.

In second regime $\alpha>1$ and $\frac{1}{2}<\delta \leq \frac{\alpha}{\alpha+1}$, note first that we may impose the constraint $A_{t} \leq t^{\frac{\alpha+1}{\alpha}}$ by Theorem $\square$ as above since

$$
P_{0}\left(A_{t} \geq t^{\frac{\alpha+1}{\alpha}}\right)=\exp \left\{-t^{1+o(1)}\right\}
$$


decays faster than the desired bound. We use (4.3) with $\rho=1-\epsilon$ to see that there is $m>0$ such that

$$
P_{0}\left(t^{1-\epsilon} \leq A_{\frac{t}{2}}, S_{\frac{t}{2}}=0\right) \geq t^{-m}
$$

for large $t$. Then, the lower bound follows by the same way as in (5.13).

Finally, in the last case $\delta \geq \frac{\alpha}{(\alpha-1)_{+}}>1$, we impose $t^{1-\epsilon} \leq A_{t} \leq t^{M}$ under which Lemma $\square$ yields

$$
p_{A_{t}}\left(0, t^{\delta}\right) \geq \exp \left\{-t^{\delta+o(1)}\right\} .
$$

Given this bound, we can argue as before to obtain

$$
\begin{aligned}
\text { R.H.S. of (5.2) } & \geq E_{0}\left[p_{A_{t}}\left(0, t^{\delta}\right): S_{t}=t^{\gamma}, t^{1-\epsilon} \leq A_{t} \leq t^{M}, S_{\frac{t}{2}}=0\right] \\
& \geq \exp \left\{-t^{\delta+o(1)}\right\} t^{-m} P_{0}\left(S_{\frac{t}{2}}=t^{\gamma}\right)-P_{0}\left(A_{t} \geq t^{M}\right) .
\end{aligned}
$$

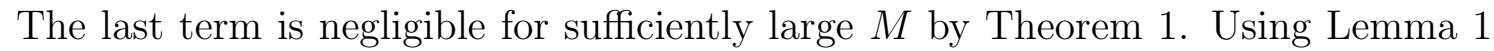
once again, we arrive at the desired lower bound.

Proof of Theorem 4 . This is an easy consequence of (4.3) and Theorem $\mathbb{0}$. Indeed, we have

R.H.S. of (5.2)

$$
\begin{aligned}
& \geq E_{0}\left[p_{A_{t}}\left(0, t^{\delta}\right): S_{t}=t^{\gamma},\left(\frac{t}{2}\right)^{2 \delta} \leq A_{t} \leq t^{\frac{\alpha+d}{\alpha}}, S_{\frac{t}{2}}=0\right] \\
& \geq \inf \left\{p_{u}\left(0, t^{\delta}\right): u \in\left[\left(\frac{t}{2}\right)^{2 \delta}, t^{\frac{\alpha+d}{\alpha}}\right]\right\} P_{0}\left(A_{\frac{t}{2}} \geq\left(\frac{t}{2}\right)^{2 \delta}, S_{\frac{t}{2}}=0\right) P_{0}\left(S_{\frac{t}{2}}=t^{\gamma}\right) \\
& \quad-P_{0}\left(A_{t} \geq t^{\frac{\alpha+d}{\alpha}}\right)
\end{aligned}
$$

as before and the last term decays super-polynomially by Theorem $\mathrm{m}$. Now if $\gamma$ and

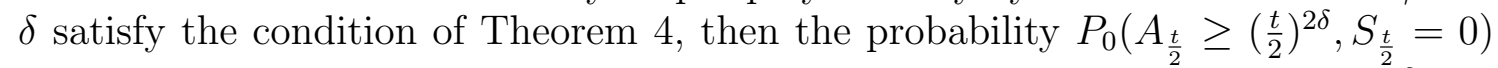
is bounded from below by a power of $t$ and the transition probabilities $p_{u}\left(0, t^{\delta}\right)$ for $u \in\left[t^{2 \delta}, t^{\frac{\alpha+d}{\alpha}}\right]$ and $P_{0}\left(S_{\frac{t}{2}}=t^{\gamma}\right)$ also decay like a negative power of $t$ by the local central limit theorem.

5.2. Proof of Theorem 5. We shall present a proof for the third regime in (2.16]) and later indicate how to adapt the argument to the other regimes, see Remark [0 below. Note that in the regimes of concern, the tail asymptotics are determined by the probability that the second ( $d$-dimensional) component of the random walk goes to an extreme point of $z$-field and stays there. In particular, the vertical displacement plays no role in the exponents. The key observation is that the heat kernel bound is violated when $z$-field has a atypically large extreme value and this has a polynomial decaying probability. 
Fix $\rho$ which appears in the lower bound of Theorem 3 and also the corresponding $\mu$ in the lower bound of Theorem $\mathbb{m}$. Consider the event

$$
\left\{\omega: \max _{|x| \leq t^{\mu-\frac{r}{2}}} z(x) \geq t^{\frac{d \mu}{\alpha}+\frac{r}{2}}\right\} .
$$

This event has a probability larger than $t^{-\frac{r(\alpha+d)}{2}+o(1)}$. On this event, we let the random walk go to a point that maximizes $z(\cdot)$ within $|x| \leq t^{\mu-\frac{r}{2}}$ and then follow the same strategy as in the lower bound of Theorem $\mathrm{m}$. This strategy gives a slightly better lower bound

$$
P_{0}^{\omega}\left(X_{t}=t^{\delta} \mathbf{e}_{1}\right) \geq \exp \left\{-t^{q(\alpha, \delta)-r+o(1)}\right\}
$$

and hence we have proved the lower bound

$$
\mathbb{P}\left(\tau_{r}>t\right) \geq t^{-\frac{r(\alpha+d)}{2}+o(1)} .
$$

Remark 10. When $d \geq 2$ and $\delta \in\left(\frac{2 \alpha}{2 \alpha+d} \vee \frac{d}{4 \alpha}, \frac{2 \alpha+d}{2 \alpha}\right]$ or $d \geq 1$ and $\delta \in\left(\frac{2 \alpha+d}{2 \alpha}, \frac{\alpha}{(\alpha-d)_{+}}\right)$ (the fourth regime), the event

$$
\max _{|x| \leq t^{\mu-\frac{r}{2}}} z(x) \geq t^{\frac{d \mu}{\alpha}+r} \text { or } \max _{|x| \leq t^{\mu-r}} z(x) \geq t^{\frac{d \mu}{\alpha}}
$$

respectively plays the role of (5.20). When $q(\alpha, \delta) \vee((2 \gamma-1) \wedge \gamma)=2 \delta-1$ (the second regime), the event

$$
\max _{|x| \leq t^{\delta-\frac{r}{2}}} z(x) \geq \begin{cases}t^{1+\delta+\frac{3 r}{2}}, & d=1 \\ t^{2(1-\delta+r)}, & d \geq 2\end{cases}
$$

plays the role of $(5 \cdot 20)$.

\section{Open PROBLEMS}

We list a few open problems:

(i) It is of course desirable to get rid of $o(1)$ errors from both Theorems $\mathrm{W}$ and $\mathrm{B}$. The proof of Theorem $\square$ tells us that it would be helpful to understand the asymptotics of $\sup _{|x| \leq R} E_{x}\left[\int_{0}^{T} z\left(S_{u}\right) \mathrm{d} u\right]$ as $R, T \rightarrow \infty$ in a coupled manner. For this problem, one should be careful about the formulation. As observed in [33], the result can fluctuate in the leading order and then the weak and almost sure limit may differ.

(ii) It is also interesting to understand how $P_{0}\left(A_{t} \geq t^{\frac{\alpha+1}{2 \alpha}} b(t)\right)(d=1, \alpha \leq 1)$ and $P_{0}\left(A_{t} \geq t^{\frac{d}{2 \alpha}} b(t)\right)\left(d \geq 2, \alpha \leq \frac{d}{2}\right)$ behave for a function $b(t) \rightarrow \infty$. This possibly leads to the law of iterated logarithm type result.

(iii) In the case $\mathbb{E}[z(0)]<\infty$, it would be nice to complement Theorem $\nabla$ by

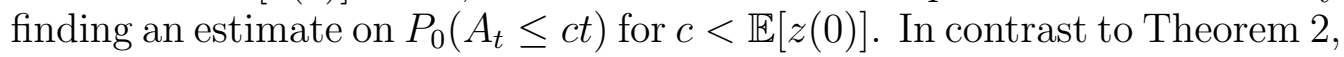
the strategy for the random walk is to stay on a low level set of $z$-field. Therefore it is natural to expect that the order of $\log P_{0}\left(A_{t} \leq c t\right)$ is different 
from Theorem 2]. The study of lower deviations is also related to the ondiagonal estimate for the random conductance model through

$$
P_{0}^{\omega}\left(X_{t}=0\right)=E_{0}\left[p_{A_{t}}(0,0): S_{t}=0\right] \sim c t^{-\frac{d}{2}} E_{0}\left[A_{t}^{-\frac{1}{2}} \mid S_{t}=0\right] .
$$

(iv) Concerning the random conductance model, a natural extension is to make other lines parallel to axis random. It no longer admits the time change representation in terms of the random walk in random scenery. When conductance is bounded and uniformly elliptic, it is what is called toy-model in [6], Section 2.3. The quenched invariance principle proved there extends to the case where the conductance of every coordinate axis has finite mean. The heavy tailed case would be much more complicated and there might be an explosion in finite time.

\section{Appendix A.}

We include the following version of Chen's result for the sake of completeness. The bound and its proof are literary taken from [12]. As it is a non-asymptotic result, it applies to $f$ which depends on $t$ and one can deduce the version indicated in Remark 6 .

Proposition 1. Suppose $t>0, f: \mathbb{Z}^{d} \rightarrow[0, \infty)$ be a bounded function and $a, b:(0, \infty) \rightarrow$ $(1, \infty)$ satisfy

$$
\sup _{x \in \operatorname{supp} f} E_{x}\left[\int_{0}^{\frac{t}{b(t)}} f\left(S_{u}\right) \mathrm{d} u\right] \leq a\left(\frac{t}{b(t)}\right)
$$

Then for $\lambda>0$,

$$
P_{0}\left(\int_{0}^{t} f\left(S_{u}\right) \mathrm{d} u \geq \lambda a\left(\frac{t}{b(t)}\right) b(t)\right) \leq 2^{\frac{1}{2}} e^{\frac{1}{24(b(t)-1)}}\left(\frac{\lambda e}{4}\right)^{-b(t)+1} .
$$

Proof. Note first that the supremum in (A.1]) can be extended to $x \in \mathbb{Z}^{d}$ by using the strong Markov property at the hitting time to $\operatorname{supp} f$. The key lemma is the following non-asymptotic bound for the moments of an additive functional.

Lemma 4. For any $m \in \mathbb{N}, t>0$ and a bounded function $f: \mathbb{Z}^{d} \rightarrow[0, \infty)$,

$$
\sup _{x \in \mathbb{Z}^{d}} E_{x}\left[\left(\int_{0}^{t} f\left(S_{u}\right) \mathrm{d} u\right)^{m}\right] \leq m ! \sup _{x \in \mathbb{Z}^{d}} E_{x}\left[\int_{0}^{t} f\left(S_{u}\right) \mathrm{d} u\right]^{m} .
$$

This goes back at least to [27], see the proof of Lemma 3 there.

In what follows, we write $[b(t)]$ for the integer part of $b(t)$ and

$$
I_{k}=\int_{\frac{(k-1) t}{[b(t)]}}^{\frac{k t}{[b(t)]}} f\left(S_{u}\right) \mathrm{d} u
$$


for $1 \leq k \leq[b(t)]$. Then by the multinomial identity,

$$
\begin{aligned}
E_{x}\left[\left(\int_{0}^{t} f\left(S_{u}\right) \mathrm{d} u\right)^{[b(t)]}\right] & =E_{x}\left[\left(\sum_{k=1}^{[b(t)]} \int_{\frac{(k-1) t}{[b(t)]}}^{\frac{k t}{[b(t)]}} f\left(S_{u}\right) \mathrm{d} u\right)^{[b(t)]}\right] \\
& \leq \sum_{k_{1}+\cdots+k_{[b(t)]}=[b(t)]} \frac{[b(t)] !}{k_{1} ! \cdots k_{[b(t)]} !} E_{x}\left[I_{1}^{k_{1}} \cdots I_{[b(t)]}^{\left.k_{[b(t)]}\right]} .\right.
\end{aligned}
$$

Applying the Markov property at $\frac{k t}{[b(t)]}(1 \leq k<[b(t)])$ and using Lemma $\mathbb{\theta}$, we find

$$
E_{x}\left[I_{1}^{k_{1}} \cdots I_{[b(t)]}^{k_{[b(t)]}}\right] \leq k_{1} ! \cdots k_{[b(t)]} ! \sup _{y \in \mathbb{Z}^{d}} E_{y}\left[\int_{0}^{\frac{t}{[b(t)]}} f\left(S_{u}\right) \mathrm{d} u\right]^{k_{1}+\cdots+k_{[b(t)]}} .
$$

Hence the right hand side of (A.5) is bounded by

$$
[b(t)] !\left(\begin{array}{c}
2[b(t)] \\
{[b(t)]}
\end{array}\right) \sup _{y \in \mathbb{Z}^{d}} E_{y}\left[\int_{0}^{\frac{t}{[b(t)]}} f\left(S_{u}\right) \mathrm{d} u\right]^{[b(t)]} \leq \frac{(2[b(t)]) !}{[b(t)] !} a\left(\frac{t}{[b(t)]}\right)^{[b(t)]} .
$$

Finally, the Markov inequality together with Stirling's approximation (see [18], Chapter II-9) yields

$$
\begin{aligned}
P_{x} & \left(\int_{0}^{t} f\left(S_{u}\right) \mathrm{d} u \geq \lambda a\left(\frac{t}{[b(t)]}\right)[b(t)]\right) \\
& \leq\left(\lambda a\left(\frac{t}{[b(t)]}\right)[b(t)]\right)^{-[b(t)]} E_{x}\left[\left(\int_{0}^{t} f\left(S_{u}\right) \mathrm{d} u\right)^{[b(t)]}\right] \\
& \leq \lambda^{-[b(t)]}[b(t)]^{-[b(t)]} \frac{\sqrt{2 \pi}(2[b(t)])^{2[b(t)]+\frac{1}{2}} e^{-2[b(t)]+\frac{1}{24[(b)]}}}{\sqrt{2 \pi}[b(t)]^{[b(t)]+\frac{1}{2}} e^{-[b(t)]}} \\
& =2^{\frac{1}{2}} e^{\frac{1}{24[b(t)]}}\left(\frac{\lambda e}{4}\right)^{-[b(t)]} .
\end{aligned}
$$

\section{ACKNOWLEDGMENTS}

The first author thanks RIMS at Kyoto university for the kind hospitality and the financial support. The second author was partially supported by JSPS KAKENHI Grant Number 24740055 and 16K05200. The authors are grateful to a reviewer for pointing out a flaw in the proof of the lower bounds in Theorem 3 and 4 .

\section{REFERENCES}

[1] S. Andres, J.-D. Deuschel, and M. Slowik. Heat kernel estimates for random walks with degenerate weights. Electron. J. Probab., 21:Paper No. 33, 21 pp., 2016. 
[2] A. Asselah and F. Castell. Large deviations for Brownian motion in a random scenery. Probab. Theory Related Fields, 126(4):497-527, 2003.

[3] A. Asselah and F. Castell. Quenched large deviations for diffusions in a random Gaussian shear flow drift. Stochastic Process. Appl., 103(1):1-29, 2003.

[4] A. Asselah and F. Castell. A note on random walk in random scenery. Ann. Inst. H. Poincaré Probab. Statist., 43(2):163-173, 2007.

[5] A. Asselah and F. Castell. Random walk in random scenery and self-intersection local times in dimensions $d \geq 5$. Probab. Theory Related Fields, 138(1-2):1-32, 2007.

[6] M. Biskup. Recent progress on the random conductance model. Probab. Surveys, 8:294-373, 2011.

[7] A. N. Borodin. A limit theorem for sums of independent random variables defined on a recurrent random walk. Dokl. Akad. Nauk SSSR, 246(4):786-787, 1979.

[8] A. N. Borodin. Limit theorems for sums of independent random variables defined on a transient random walk. Zap. Nauchn. Sem. Leningrad. Otdel. Mat. Inst. Steklov. (LOMI), 85:17-29, 237, 244, 1979. Investigations in the theory of probability distributions, IV.

[9] S. Boucheron, G. Lugosi, and P. Massart. Concentration inequalities. Oxford University Press, Oxford, 2013. A nonasymptotic theory of independence, With a foreword by Michel Ledoux.

[10] F. Castell. Moderate deviations for diffusions in a random Gaussian shear flow drift. Ann. Inst. H. Poincaré Probab. Statist., 40(3):337-366, 2004.

[11] F. Castell and F. Pradeilles. Annealed large deviations for diffusions in a random Gaussian shear flow drift. Stochastic Process. Appl., 94(2):171-197, 2001.

[12] X. Chen. Moderate deviations for Markovian occupation times. Stochastic Process. Appl., 94(1):51-70, 2001.

[13] X. Chen. Random walk intersections, volume 157 of Mathematical Surveys and Monographs. American Mathematical Society, Providence, RI, 2010. Large deviations and related topics.

[14] E. Csáki, W. König, and Z. Shi. An embedding for the Kesten-Spitzer random walk in random scenery. Stochastic Process. Appl., 82(2):283-292, 1999.

[15] D. A. Darling and M. Kac. On occupation times for Markoff processes. Trans. Amer. Math. Soc., 84:444-458, 1957.

[16] T. Delmotte and J.-D. Deuschel. On estimating the derivatives of symmetric diffusions in stationary random environment, with applications to $\nabla \phi$ interface model. Probab. Theory Related Fields, 133(3):358-390, 2005.

[17] F. den Hollander and J. E. Steif. Random walk in random scenery: a survey of some recent results. In Dynamics $\mathcal{E}$ stochastics, volume 48 of IMS Lecture Notes Monogr. Ser., pages 53-65. Inst. Math. Statist., Beachwood, OH, 2006.

[18] W. Feller. An introduction to probability theory and its applications. Vol. I. Third edition. John Wiley \& Sons, Inc., New York-London-Sydney, 1968.

[19] K. Fleischmann, P. Mörters, and V. Wachtel. Moderate deviations for a random walk in random scenery. Stochastic Process. Appl., 118(10):1768-1802, 2008.

[20] N. Gantert, W. König, and Z. Shi. Annealed deviations of random walk in random scenery. Ann. Inst. H. Poincaré Probab. Statist., 43(1):47-76, 2007.

[21] N. Gantert, R. van der Hofstad, and W. König. Deviations of a random walk in a random scenery with stretched exponential tails. Stochastic Process. Appl., 116(3):480-492, 2006.

[22] J. Gärtner and W. König. Moment asymptotics for the continuous parabolic Anderson model. Ann. Appl. Probab., 10(1):192-217, 2000.

[23] N. Guillotin-Plantard. Large deviations for a Markov chain in a random landscape. Adv. in Appl. Probab., 34(2):375-393, 2002. 
[24] N. Guillotin-Plantard, J. Poisat, and R. S. dos Santos. A quenched functional central limit theorem for planar random walks in random sceneries. Electron. Commun. Probab., 19:no. 3, 9, 2014.

[25] S. Kakutani. Random ergodic theorems and Markoff processes with a stable distribution. In Proceedings of the Second Berkeley Symposium on Mathematical Statistics and Probability, 1950, pages 247-261. University of California Press, Berkeley and Los Angeles, 1951.

[26] H. Kesten and F. Spitzer. A limit theorem related to a new class of self-similar processes. $Z$. Wahrsch. Verw. Gebiete, 50(1):5-25, 1979.

[27] R. Khas'minskii. On positive solutions of the equation $\mathfrak{A} u+v u=0$. Theory Probab. Appl., $4(3): 309-318,1959$.

[28] D. Khoshnevisan and T. M. Lewis. A law of the iterated logarithm for stable processes in random scenery. Stochastic Process. Appl., 74(1):89-121, 1998.

[29] W. König. Upper tails of self-intersection local times of random walks: survey of proof techniques. Actes des rencontres du CIRM: Excess Self-Intersections and Related Topics, (1):15-24, 2010.

[30] Y. Li. Moderate deviations for stable random walks in random scenery. J. Appl. Probab., 49(1):280-294, 2012.

[31] B. Remillard. Large deviations estimates for occupation time integrals of Brownian motion. In Stochastic models (Ottawa, ON, 1998), volume 26 of CMS Conf. Proc., pages 375-398. Amer. Math. Soc., Providence, RI, 2000.

[32] M. Talagrand. A new look at independence. Ann. Probab., 24(1):1-34, 1996.

[33] R. van der Hofstad, P. Mörters, and N. Sidorova. Weak and almost sure limits for the parabolic Anderson model with heavy tailed potentials. Ann. Appl. Probab., 18(6):2450-2494, 2008.

[34] L. Zhang. Strong approximation for the general Kesten-Spitzer random walk in independent random scenery. Sci. China Ser. A, 44(5):619-630, 2001.

(Jean-Dominique Deuschel) Institut für Mathematik, Technische Universität Berlin, Berlin, Germany and Research Institute in Mathematical Sciences, Kyoto UniverSITY, KYOTO, JAPAN

E-mail address: deuschel@math.tu-berlin.de

(Ryoki Fukushima) Research Institute in Mathematical Sciences, Kyoto University, KYOTO, JAPAN

E-mail address: ryoki@kurims.kyoto-u.ac.jp 